\title{
Pilot-scale biofiltration at a materials recovery facility: The impact on bioaerosol control
}

\author{
I.E. Ibanga $^{a}$, L.A. Fletcher ${ }^{\text {a,* }}$, C.J. Noakes ${ }^{a}$, M.F. King ${ }^{a}$, D. Steinberg ${ }^{b}$ \\ a School of Civil Engineering, University of Leeds, Woodhouse Lane, Leeds LS2 9JT, UK \\ ${ }^{\mathrm{b}}$ Associated Waste Management Limited, St Bernard's Mill, Gelderd Road, Leeds LS27 7NA, UK
}

\section{A R T I C L E I N F O}

\section{Article history:}

Received 4 April 2018

Revised 1 September 2018

Accepted 4 September 2018

\section{Keywords:}

Biofilter

Bioaerosols

Odour

Waste management

Woodchips

\begin{abstract}
A B S T R A C T
This study investigated the performance of four pilot-scale biofilters for the removal of bioaerosols from waste airstreams in a materials recovery facility (MRF) based in Leeds, UK. A six-stage Andersen sampler was used to measure the concentrations of four groups of bioaerosols (Aspergillus fumigatus, total fungi, total mesophilic bacteria and Gram negative bacteria) in the airstream before and after passing through the biofilters over a period of 11 months. The biofilters achieved average removal efficiency (RE) of $70 \%$ (35 to $97 \%$ ) for $A$. fumigatus, 71\% (35 to 94\%) for total fungi, 68\% (47 to 86\%) for total mesophilic bacteria and $50 \%$ ( -4 to $85 \%$ ) for Gram negative bacteria, provided that the inlet concentration was high $\left(10^{3}-10^{5}-\right.$ $\mathrm{cfu} \mathrm{m}^{-3}$ ), which is the case for most waste treatment facilities. The performance was highly variable at low inlet concentration with some cases showing an increase in outlet concentrations, suggesting that biofilters had the potential to be net emitters of bioaerosols. The gas phase residence time did not appear to have any statistically significant impact on bioaerosol removal efficiency. Particle size distribution varied between the inlet and outlet air, with the outlet having a greater proportion of smaller sized particles that represent a greater human health risk as they can penetrate deep into the respiratory system where gaseous exchange occurs. However, the outlet concentrations were low and would further be diluted by wind in full scale applications. In conclusion, this study shows that biofilters designed and operated for odour degradation can also achieve significant bioaerosol control in waste gas.
\end{abstract}

(c) 2018 Elsevier Ltd. All rights reserved.

\section{Introduction}

With continuous emphasis on meeting the landfill diversion targets in the UK as established in the Landfill Directive (1999/31/EC) and with the launch of the Landfill Allowance Trading Scheme (LATS) in 2004 (Calaf-Forn et al., 2014), there has been an increase in the number of waste management facilities (Stagg et al., 2010; Environment Agency, 2017). Some of these facilities are enclosed, and can include mechanical biological treatment (MBT), in-vessel composting (IVC), anaerobic digestion (AD) and materials recovery facilities (MRF) or combinations of different waste management systems. These facilities, while achieving cutting edge recycling performance and value recovery from waste streams, have the potential for air pollution within the facility and externally via their extract ventilation especially due to odour and bioaerosol emissions.

Bioaerosols, which comprise predominantly plant pollen, microorganisms (viable or non-viable) and/or microbial

\footnotetext{
* Corresponding author.

E-mail address: l.a.fletcher@leeds.ac.uk (L.A. Fletcher).
}

metabolites, have the potential to cause health problems in exposed persons with symptoms such as irritation of the respiratory tract and eyes, coughing, wheezing, tiredness, rashes on skin, diarrhoea, asthma, headache, allergic rhinitis and hypersensitivity pneumonitis (Husman, 1996; Menetrez et al., 2009). Studies show that bioaerosol exposure can cause ill-health in exposed population (Douwes et al., 2003; Searl, 2008; Pearson et al., 2015) Lower forced vital capacity was reported in exposed compost workers $(n=190)$ than in controls $(n=38)$ (van Kampen et al., 2012). Hambach et al. (2012), while assessing work-related health symptoms among compost workers, reported elevated proportion of exposed group $(n=31)$ presenting with respiratory symptoms (29.0\%), eye, nose and throat irritation symptoms (35.5\%), gastrointestinal symptoms (29.0\%) and skin rashes $(20.0 \%)$ as against the control group $(n=31)$ who showed $3.3 \%, 13.3 \%, 6.7 \%$ and $0.0 \%$, respectively, for these symptoms. The risk of waste workers' exposure to bioaersols may be dependent on the work task (mostly indoors for enclosed facilities), their proximity to the source of bioaerosols and the abatement system being used on site (Stagg et al., 2010). 
In the UK, the Environment Agency (EA) is responsible for regulating waste management facilities, usually done through the granting of Permits to Operate. Part of the EA's remit is to ensure that odours and bioaerosols do not adversely impact the surrounding population (Frederickson et al., 2013), and so have included bioaerosol monitoring requirements as an environmental permit condition, and to assess the performance of abatement systems at operation in such facilities (Environment Agency, 2017). The EA gave a precautionary guidance for composting operators when applying for operating permits. This guidance stipulates that concentrations of bioaerosols (as predicted or measured directly) need to be maintained no higher than acceptable levels at $250 \mathrm{~m}$ from the composting site or the nearest sensitive receptor (such as a dwelling or workplace which is not part of the composting site), whichever is closer (Environment Agency, 2010). These acceptable levels have been defined as $500 \mathrm{cfu} \mathrm{m}^{-3}, 1000 \mathrm{cfu} \mathrm{m}^{-3}$ and $300 \mathrm{cfu} \mathrm{m}^{-3}$ for Aspergillus fumigatus, total bacteria and Gramnegative bacteria, respectively, as measured by the standardised monitoring protocol (i.e. the AfOR protocol later replaced in 2017 by the M9 protocol). However, an updated regulatory position statement (RPS) on monitoring bioaerosols at regulated facilities was provided by the EA in January 2018, and excluded the reporting of Gram-negative bacteria (Environment Agency, 2018). In the UK, there are no regulatory occupational limits for bioaersols as the acceptable levels stated above are not based on dose-response relationships (Pearson et al., 2015). However, the Control of Substances Hazardous to Health (COSHH) Regulation issued by the Health and Safety Executive (HSE) provides employers with the requirements for assessing, monitoring and controlling the exposure of employees to hazardous substances at work environments (HSE, 2013), and thus, applies to workers in waste handling facilities. In Germany, there is a regulatory occupational limit of 50 $000 \mathrm{cfu} \mathrm{m}^{-3}$ for mesophilic fungi (including A. fumigatus) in breathable air within the workplace (BAUA, 2013 cited in Pearson et al., 2015).

Biofilters have been used as an abatement method in the waste management industry for many years with varying degrees of success. Biofilters are three phase bioreactors (gas, liquid, solid) composed of filter beds which have high porosity; high buffer capacity; high nutrient availability and high moisture retention capacity which altogether provide suitable internal environments that support the growth and attachment of a mixed-culture of pollutantdegrading microorganisms (Elias et al., 2002; Dastous et al., 2005). Biofilters offer a cost-efficient and potentially environmentally friendly alternative to traditional air treatment technologies, particularly for odour and gas treatment because of the low energy requirement; relatively low construction cost; no generation of secondary pollutants that require subsequent disposal; and capacity to treat a broad spectrum of gaseous compounds (Devinny et al., 1999; Fulazzaky et al., 2014). Biofilters are a method of biological air treatment systems that utilise populations of microorganisms to convert certain organic and inorganic pollutants into compounds and/or forms that are less toxic and/or odourless. The microbial population, which may be dominated by a single species or be composed of different interacting species, employ oxidative, and sometimes, reductive reactions to convert the airborne pollutants into $\mathrm{CO}_{2}$, water vapour, and to increase their population using these pollutants as energy and carbon sources (Fletcher et al., 2014). The design and operation of the early biofilter systems were based on a very basic understanding of their method of operation. Although in recent years the structural materials used for biofilters have become more sophisticated, and in the UK there is a move towards using emission stacks, the fundamental design criteria have changed very little (Fletcher et al., 2014).

Several studies have been carried out over the past two decades, in an attempt to better understand the principles of biofilter design and operation to achieve significant odour and bioaerosol removal. Some of these have looked at the microbiology of the biofilters (Juteau et al., 1999), technical characteristics(Pagella and De Faveri, 2000), performance (Jorio et al., 2000), modelling (Alonso et al., 1999), and economic viability (Gao et al., 2001). It is acknowledged that biofilters offer a versatile and cost effective option for the management of contaminated air from waste handling and treatment facilities (Devinny et al., 1999; Kummer and Thiel, 2008; Frederickson et al., 2013). However, there is a lot of contradictory data and many gaps in the knowledge which need to be addressed if biofilters are to be designed to effectively control all emissions and to perform efficiently. In particular several authors have suggested that media characteristics such as porosity, moisture content, nutrient content, temperature and water retention capacity are the most important factors governing biofilter performance, although the optimum ranges quoted in the literature vary significantly from one author to another (Devinny et al., 1999; Nicolai and Janni, 2001a; Quigley et al., 2004; Schlegelmilch et al., 2005; Álvarez-Hornos et al., 2008; Frederickson et al., 2013). Other authors suggest that operating parameters such as empty bed residence time (EBRT), contaminant loading rate and upflow or downflow configuration are important factors but again there seems to be little consensus as to what the optimum ranges are (Leson and Winer, 1991; Lu et al., 2002; Chen and Hoff, 2009; Liu et al., 2009).

Recent studies by Frederickson et al. (2013) and Fletcher et al. (2014) have evaluated the performance of laboratory-scale and full-scale biofilters in terms of their capacity for simultaneous control of odour and bioaerosols by considering what parameters were vital in defining what design, conditions and maintenance schedules were required for optimum performance. However, these studies concluded that the literature contains apparently contradictory information regarding the impact of biofilter design and operating parameters (such as empty bed residence time, moisture content, media $\mathrm{pH}$ and temperature) on odour and bioaerosol emissions and removal. This is a major issue for waste management operators and regulators as there is no clear guidance in terms of design and operating parameters that would provide a robust evidence base against which to benchmark the effectiveness of existing biofilters and future abatement system proposals including biofilters. Although bioaersosols removal mechanisms by biofilter have been thought to include inertial deposition, diffusional (or Brownian) deposition and flow line interception (Ottengraf and Konings, 1991), Frederickson et al. (2013) recommended that further research is required to determine the relationship between odour and bioaerosol emissions from biofilters to determine the extent to which biofilters may be used to effectively reduce both odour and bioaerosols, and to identify best practice techniques for optimising biofilters to maximise control of both odour and bioaerosol emissions. This is especially necessary because of the differences in the removal mechanisms of odour and bioaerosols. Literature suggests that odour removal mechanisms is dependent on sorption of the odorous compounds into the biofilm layer on the media surface where biodegradation takes place, a function which relies on long residence time; whereas bioaerosol removal is achieved via particle impaction onto the media partcles, and so an extended residence time may not impact positively on removal (Devinny et al., 1999; Fletcher et al., 2014). Thus, it is imperative to develop a better understanding of biofilter design and effective performance monitoring techniques especially if they are to continue to control all emissions and achieve their full potential.

This study was aimed at investigating the performance of pilotscale biofilters for removal of bioaerosols from waste airstreams from a materials recovery facility (MRF) which acted as a source of bioaerosols. The objectives of this research were: (1) to assess 
the impact of empty bed residence time (EBRT) on the performance of pilot-scale biofilters in terms of bioaerosol reductions; $(2)$ to evaluate the net bioaerosol emitting potentials of biofilters and to assess the effect of inlet concentration on bioaerosol control; and (3) to assess size distribution of bioaerosol particles in biofilter exhausted air and to relate these to the tidal volume inhaled by humans. To achieve these objectives, four groups of bioaerosols were measured including Aspergillus fumigatus, total fungi, total mesophilic bacteria and Gram negative bacteria. The choice of these microorganisms was informed by the need to reflect the range covered in the Sniffer report (ER36) on understanding biofilter performance and determining emission concentrations under operational conditions (Fletcher et al., 2014) as well as those specified in the Technical Guidance Note (M9) for monitoring of bioaerosols at regulated facilities (Environment Agency, 2017). Bioaerosol concentrations were measured in the airstream before (inlet) and after (outlet) passing through the biofilters over a period of 11 months. The inlet and outlet concentrations were compared to evaluate removal efficiencies, and these were also compared with the background concentrations measured upwind of the facility. The measurements were carried out using a sixstage Andersen sampler to obtain particle size distributions for each of the four bioaerosol groups. This paper also comments on the variability in the data and the benefits and limitations of using pilot scale approaches.

\section{Material and methods}

\subsection{Description of pilot study site}

The pilot study was conducted at an enclosed MRF located in Leeds, UK. The building dimensions were $100 \mathrm{~m} \times 40 \mathrm{~m} \times 15 \mathrm{~m}$ to the apex, giving a total volume of $60,000 \mathrm{~m}^{3}$. The facility handles approximately 200,000 tonnes of household waste per year from around 250,000 households across Leeds, Bradford and Calderdale (Holland, 2011). This site was chosen because of the potential for significant odour and bioaerosol emissions. Stagg et al. (2013), in their study, stated that exposure to microorganisms (bacteria and fungi) in MRFs were considered medium level (between $10^{4}$ and $10^{5} \mathrm{cfu} \mathrm{m}^{-3}$ ) and occasionally showed higher levels similar to those of animal houses at $>10^{5} \mathrm{cfu} \mathrm{m}^{-3}$, and with identified species including A. fumigatus which is a known allergen. Hence, this site meets the requirement of providing air contaminated with significant levels of bioaerosols required to test the control of bioaerosol emissions in this study.

The plant is enclosed with a large waste reception area, three hand-picking stations, a baling area and various bays for collection of woods, electrical materials, plastics, metals, paper and bricks. It combines mechanized and hand-sorting techniques which agitate the waste, potentially releasing high concentrations of bioaerosols and odorous volatiles. Various vehicle types are operated during the working hours (07:30 and 18:30 daily from Mondays to Fridays, and 08:00 to 13:00 on Saturdays) including, forklift trucks, dinosaurus shredders, front loaders and waste trucks.

At the time of this study, the plant had 11 Modular air filtration units containing pre-filters and impregnated activated carbon cartridges for dust and odour removal, respectively, which recirculate air within the building. Together, the units delivered 2.64 air changes per hour (surpassing the recommended industry average of 1.5 air changes per hour), treating a total air volume of $158,400 \mathrm{~m}^{3}$ per hour (Varley, 2013). This was done following expert recommendation to allow for an increased factor of safety and increased negative pressure to contain any fugitive emissions.

\subsection{Description of Pilot-scale biofilter system}

The pilot-scale biofilter (BF) system (Fig. 1 a) was designed to meet odour treatment specifications as recommended by Fletcher et al. (2014). This pilot system was adopted from the design of Chen and Hoff (2012), and shows some of the key features of a full-scale system as described by Janni et al. (2011)and Fletcher et al. (2014). The system is composed of four vertical up-flow

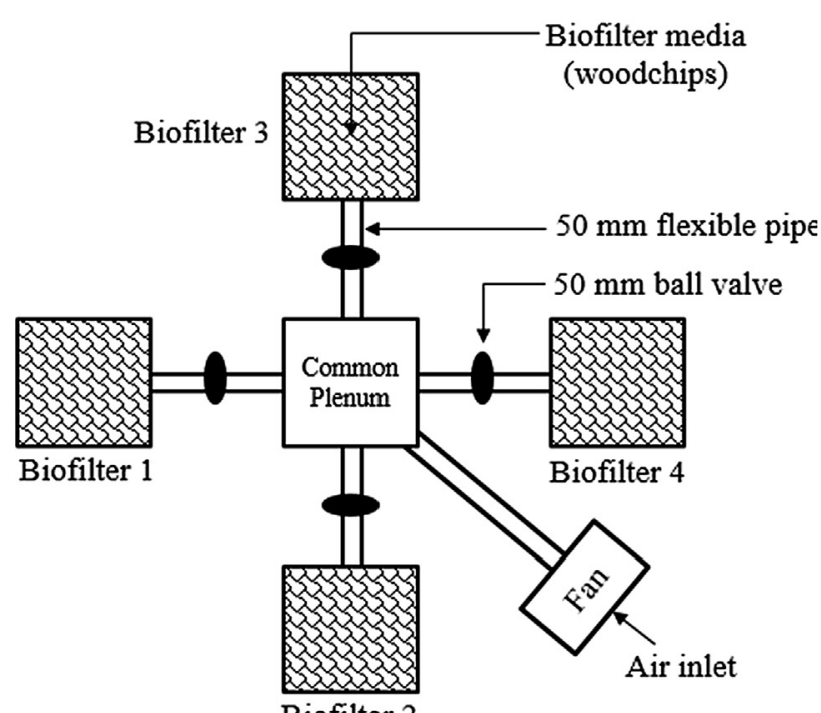

Biofilter 2

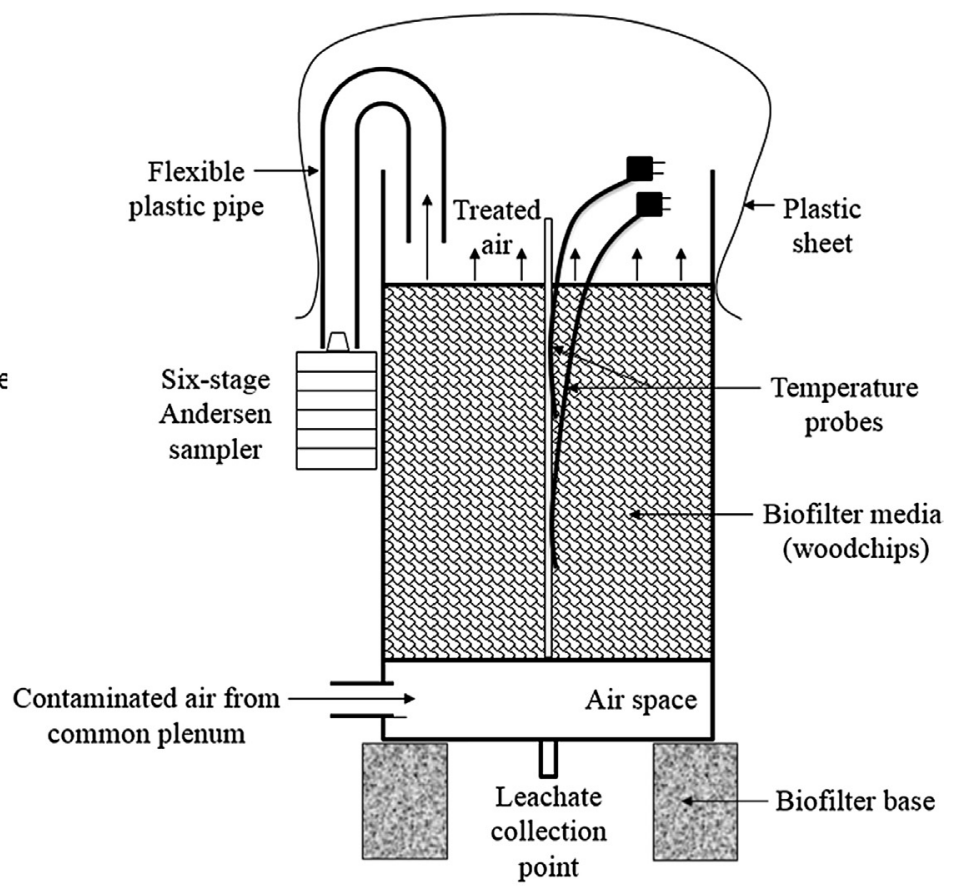

(b)

Fig. 1. Pilot-scale biofiltration system (a) schematic of four pilot-scale bioreactors and (b) schematic of each reactor with media depth of $0.5 \mathrm{~m}$. 
plastic reactors filled with wood chips as media (each reactor with length: $66 \mathrm{~cm}$, breath: $55 \mathrm{~cm}$, depth: $99 \mathrm{~cm}$ ) connected to a common plenum. Each reactor (Fig. $1 \mathrm{~b}$ ) had a $20 \mathrm{~cm}$ air-space at the bottom, with a $50 \mathrm{~cm}$ biofilter media depth (giving a total biofilter media volume of $181.5 \mathrm{~L}$ ) located above the air-space (for air distribution) separated by a metal mesh which supports the media. All four reactors were connected to a common plenum by means of $50 \mathrm{~mm}$ flexible polyvinyl chloride (PVC) pipes. A high velocity centrifugal fan was used to pump contaminated air from the waste hall into the plenum, from where each biofilter was air-fed. Lee and Lin (2007) noted that this type of fan had the capacity to handle dirtier air streams with higher system resistance. Airflow into each biofilter was measured using the a balometer capture hood (EBT731), and regulated by means of $50 \mathrm{~mm}$ ball valves to the average of three levels of empty bed residence time tested in this study - $11 \mathrm{~s}, 16 \mathrm{~s}$ and $70 \mathrm{~s}$ corresponding to flowrates of $16.5 \mathrm{~L} \mathrm{~s}^{-1}, 11.3 \mathrm{~L} \mathrm{~s}^{-1}$ and $2.6 \mathrm{~L} \mathrm{~s}^{-1}$, respectively. Water was supplied to the top of each biofilter with a combination of manual watering and an automatic irrigation system connected to a peristaltic pump and socket timer. Irrigation was controlled by the look and feel method suggested by Janni et al. (2011) whereby moisture levels were monitored to ensure dampness across $1 / 2$ to $3 / 4$ way through the media depth. Leachate from each biofilter was collected once a week for the study period; leachate $\mathrm{pH}$ was measured using a digital calibrated $\mathrm{pH}$-meter.

\subsection{Biofilter operation}

The biofilter system was operated for 11 months from May 2016 to March 2017. A total of 16 sampling visits were completed; visits 1-6 (summer 2016) and 13-16 (winter 2017) where conducted inside the building while visits 7-12 (winter 2016) were conducted outside the building. Before sampling commenced, the media in each reactor was allowed to stabilise for four weeks fol-
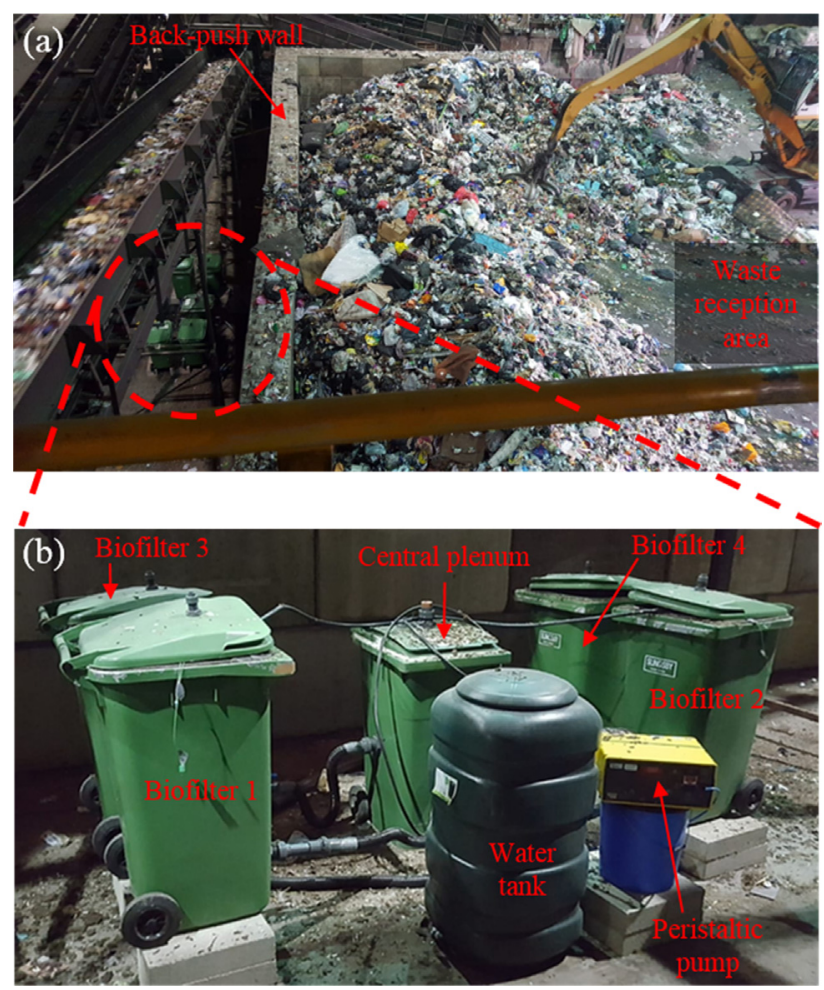

Fig. 2. (a) Location of the pilot-scale biofiltration system in the waste hall, and (b) the arrangement of the reactors behind the back-push wall. lowing recommendations in literature (Cabrol et al., 2012; Ralebitso-Senior et al., 2012). To assess the impact of EBRT on bioaerosol removal, the system was set up inside the facility just behind the back-push wall in the waste reception area (Fig. 2 a \& b). This location was selected to place the biofiltration system as close as possible to waste reception hall (thereby ensuring constant supply of air contaminated with bioaersols generated from agitation of the waste heap) without exposing the researcher to the hazards and risks associated with tipping and loading operations as well as moving vehicles within the waste hall. The indoor location was also chosen to contain any possible emissions from the biofilters especially as negative air pressure was maintained within the waste hall.

One major concern with biofilters is their potential to act as net emitters of bioaerosols at low inlet concentrations due to extra contamination by the filtration process (Ottengraf and Konings, 1991). To investigate this, the biofilters were relocated outside the waste hall to an external bay (previously used to collect fines - soils, glass, small wood, small stones, ferrous and non-ferrous materials $-0<10 \mathrm{~mm}$ ) during visits $7-12$. This location simulated ambient conditions as the biofilters were fed with air that had relatively lower concentrations of bioaerosols. The understanding was that biofilters would be considered net emitters if outlet concentrations were higher than inlet concentrations.

The biofilters were randomly selected to operate at the tested EBRT during which average moisture content of $64.7 \%$ ( 40.2 to $70.3 \%$ ), 62.4 (38.8 to $70.3 \%$ ), $55.2 \%$ (43.3 to $68.9 \%$ ) and $59.2 \%$ (41.2 to $70.5 \%$ ) were maintained in BF1, BF2, BF3 and BF4, respectively, all within the range recommended by Janni et al. (2011). In order to avoid media compaction and clogging, which could lead to the formation of preferential flow paths for air, the media was mixed with a shovel once every three weeks on days other than the sampling days (Sanchez-Monedero et al., 2003). For each sampling visit, inlet concentrations corresponded to bioaerosol samples taken from the common plenum; this was considered representative of the concentrations delivered directly to each biofilter. The outlet concentrations from each biofilter was taken from the top of each biofilter. In order to ensure the integrity of samples taken, all outlet measurements were conducted using methods which isolated treated air exiting the biofilters from the effects of ambient contamination within the waste hall. This was done by completely covering the outlet (open) end of the biofilters using plastic sheets (Fletcher et al., 2014). The biofilters were covered between sampling days to prevent surface contamination, during which treated air was released through $20 \mathrm{~mm}$ exhaust provided at the top of each biofilter cover. On sampling days, it was assumed that the headspace air was the treated air, isolated from ambient contamination and so sampling was done immediately after sheeting the biofilters. To assess whether outlet bioaerosol concentrations were comparable to the background levels, bioaerosol concentrations were measured upwind (i.e. outdoors just at the boundary of the site) at a height of $1.8 \mathrm{~m}$ above the ground (Environment Agency, 2017). Stagg et al. (2013) reported that the concentration of bacteria and fungi within MRFs were ten times the upper levels measured in ambient air. Thus, upwind (background) sampling was necessary to give information on the concentration of bioaerosols in the air blowing onto to the site (Environment Agency, 2017) which would then form the basis to assess biofilter performance in terms of achieving background (ambient) concentrations.

\subsection{Biofilter media selection and characterisation}

Based on the study by Fletcher et al. (2014), it was decided that woodchip be used as biofilter media for this study because it is easily available and can be sourced locally; relatively cost effective; 
Table 1

Characteristics of wood chips used for this study.

\begin{tabular}{|c|c|c|c|c|}
\hline \multirow[t]{2}{*}{ Characteristics } & \multirow[t]{2}{*}{ Units } & \multirow[t]{2}{*}{ Values for this study } & \multicolumn{2}{|c|}{ Values for Kafle et al. (2015) } \\
\hline & & & MWB & SWB \\
\hline Density & $\mathrm{kg} / \mathrm{m}^{3}$ & 225 & 244.3 & 200.8 \\
\hline Porosity & $\%$ & 61.4 & 59.9 & 68.4 \\
\hline Water holding capacity & g/g dry weight & 1.16 & 0.84 & 1.58 \\
\hline Moisture content & \% (wet basis) & 30 & 11 & 14 \\
\hline
\end{tabular}

MWB - Medium wood bark; SWB - Shredded wood bark.

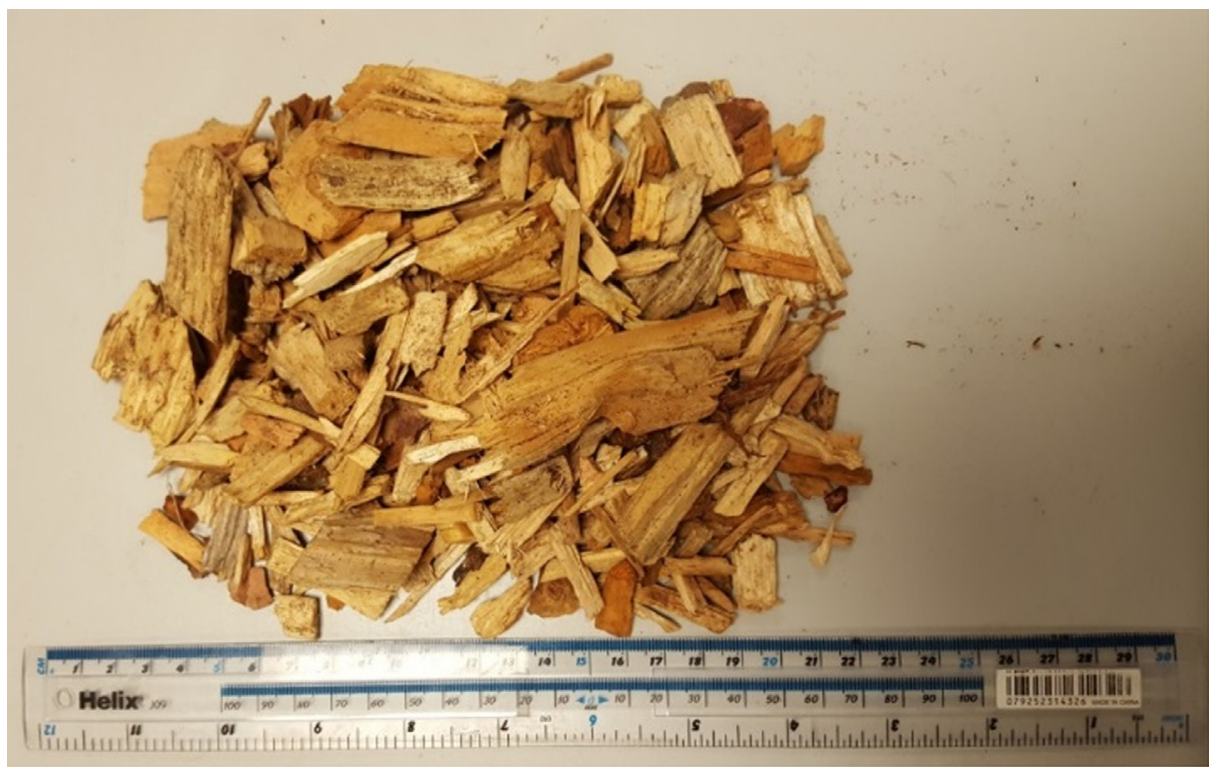

Fig. 3. Woodchips oversize fractions used for this study.

and has inherent content of nutrients (Devinny et al., 1999) and naturally harbours microbial population (Hellenbrand and Reade, 1992; Tymczyna et al., 2011); thus, eliminating the need for nutrient supply and microbial inoculation, respectively. The woodchips were purchased from a local market (Garforth Log Supplies, Peckfield House Farm, Garforth, Leeds, UK). Preliminary laboratory tests were conducted to determine the woodchip characteristics including appropriate sizing, moisture content (MC), water holding capacity (WHC), porosity and bulk density (Table 1 ).

The woodchip (as-received) was sized by sieving using the Retsch AS200 Analytical Sieve Shaker operated at an amplitude of 60 and a vibration height of $1.8 \mathrm{~mm}$ for three minutes. Sieve mesh size of $4.75 \mathrm{~mm}$ was used to obtain oversize fractions (Fig. 3) used in this study. The media MC was determined using the oven drying method which entails computing the weight loss following oven drying overnight at $105^{\circ} \mathrm{C}$ while WHC was determined by soaking the woodchips in water for $24 \mathrm{~h}$ followed by oven-drying the woodchip samples for $48 \mathrm{~h}$ at a temperature of $105{ }^{\circ} \mathrm{C}$ (Kafle et al., 2015). Media porosity (voids) was determined by the Bucket Method (Nicolai and Janni, 2001b) and bulk density was determined following the method of Valter Francescato et al. (2008).

\subsection{Air sampling and microbiological analysis}

In this study, a six-stage Andersen sampler was used to collect air samples at the various sampling points. The choice of this sampler was informed by the need to obtain both concentration and particle size data as with the study by Stagg et al. (2010). Bioaerosols detection and quantification were achieved by selective agar and visual identification. The microorganism category, specific agar type, supplements added, incubation temperatures and times for the bioaerosols are shown in Table 2.

A total of 16 sampling visits were completed, during which the six-stage Andersen sampler was used to collect air samples at the inlet (common plenum) and outlet of each biofilter for each of the microorganism categories of interest. Two replicate samples were collected at each point for each of the bioaerosol groups studied. Air was pumped through the sampler at a rate of $28.3 \mathrm{~L} \mathrm{~min}^{-1}$ with a sampling time of $1 \mathrm{~min}$ to avoid overloading the Petri dishes containing the selective media for the bioaerosols. The Environment Agency (2017) recommended that sampling time should reflect the likelihood of overloading plates ( $>300$ colonies). Preliminary sampling on this site indicated plate overload even with sampling times of 3 to $5 \mathrm{~min}$; hence, the decision for further reduction to $1 \mathrm{~min}$. Bioaerosol concentrations are known to fluctuate dramatically within a short time (Searl, 2008), and also depending on the activities within the waste hall (Stagg et al., 2013). Thus, the results of this study should be interpreted with caution as there may be uncertainties in the representativeness of the measured concentrations relative to actual exposure conditions due to periodic differences in activities. Moreover, it is estimated that $<10 \%$ of bioaerosols are viable (Blomquist, 1994; Swan et al., 2003); thus, there may be an underestimation of actual concentrations. After the incubation period, the number of colonies were counted. A positive-hole correction was done to adjust colony counts in accordance with the recommendations of Macher (1989). The results were expressed as means of duplicate samples taken in colony forming units per cubic metre of air $\left(\mathrm{cfu} \mathrm{m}^{-3}\right)$. The limit of detection of the sampler was less than $10^{2} \mathrm{cfu} \mathrm{m}^{-3}$. 
Table 2

Incubating conditions for specific bioaerosols tested.

\begin{tabular}{|c|c|c|c|c|}
\hline Bioaerosol Group & Agar & Supplements & Incubation Temperature & Incubation Time \\
\hline Aspergillus fumigatus & $20 \mathrm{~g} \mathrm{~L}^{-1}$ each of malt extract agar and bacteriological agar & $\begin{array}{l}\text { Streptomycin, } 50 \mathrm{mg} \mathrm{L}^{-1} \text {; } \\
\text { Novobiocin, } 10 \mathrm{mg} \mathrm{L}^{-1}\end{array}$ & $40^{\circ} \mathrm{C}$ & $48 \mathrm{~h}$ \\
\hline Total fungi & $20 \mathrm{~g} \mathrm{~L}^{-1}$ each of malt extract agar and bacteriological agar & $\begin{array}{l}\text { Streptomycin, } 50 \mathrm{mg} \mathrm{L}^{-1} \text {; } \\
\text { Novobiocin, } 10 \mathrm{mg} \mathrm{L}^{-1}\end{array}$ & $40^{\circ} \mathrm{C}$ & $48 \mathrm{~h}$ \\
\hline Total mesophilic bacteria & $14 \mathrm{~g} \mathrm{~L}^{-1}$ nutrient agar and $10 \mathrm{~g} \mathrm{~L}^{-1}$ bacteriological agar & Cycloheximide, $100 \mathrm{mg} \mathrm{L}^{-1}$ & $37^{\circ} \mathrm{C}$ & $48 \mathrm{~h}$ \\
\hline Gram negative bacteria & $52 \mathrm{~g} \mathrm{~L}^{-1}$ Mac Conkey agar No 1 & Cycloheximide, $200 \mathrm{mg} \mathrm{L}^{-1}$ & $37^{\circ} \mathrm{C}$ in the dark & 3-7 days \\
\hline
\end{tabular}

\subsection{Biofilter performance evaluation}

The performance of the biofilters was evaluated on the basis of removal efficiency ( $R E$ in \%) calculated using the following equation:

$R E=\left(\frac{C_{\text {in }}-C_{\text {out }}}{C_{\text {in }}}\right) \times 100$

where, Cin: inlet bioaerosol concentration; Cout: outlet bioaerosol concentration. The air sampler design also allowed for size distribution of the collected bioaerosols according to their aerodynamic behaviour. This was obtained by summing up the corrected colony counts on each stage of the sampler and grouping according to the manufacturer's aerodynamic information for stages 1 (sampler inlet) to 6 (sampler outlet) as $7.0,4.7,3.3,2.1,1.1$ and $0.65 \mu \mathrm{m}$, respectively.

\subsection{Data analysis}

All statistical analysis were carried out in the IBM SPSS Statistics for Windows (Released 2015. Version 23.0. Armonk, NY: IBM Corp., USA) and graphs generated using Origin (OriginLab, Northampton, MA, USA). Table 3 presents a summary of mean counts and standard deviations of measured concentrations of bioaersols. Normality of bioaerosol concentrations was assessed using the ShapiroWilk test. All statistics were carried out on original bioaerosol concentrations rather than the calculated RE. Differences in mean bioaerosol concentration for the background, BF inlet and all BF outlets were assessed using the one-ANOVA/Welch ANOVA, regardless of whether or not the assumption of normality was met. In all cases, the assumption of homogeneity of variances was violated, as assessed by Levene's test for equality of variances $(p<0.05)$ for all groups of bioaerosols.

For visits 1-6, Welch ANOVA followed by Games-Howell post hoc analysis indicated statistically significant differences $(p<0.05)$ between the inlet samples and all outlet samples as well as background concentration of $A$. fumigatus, total fungi and total mesophilic bacteria. There was no statistically significant difference between the inlet and outlet concentration of Gram negative bacteria ( $p=.178$ ). For visits $7-12$, there was no statistically significant difference between the mean concentrations of background, inlet and all outlets samples of A. fumigatus $(p=0.054)$ and Gram negative bacteria ( $p=0.776$ ) as assessed by Welch ANOVA. However, Games-Howell post hoc analysis showed statistically significant differences between the inlet concentration and outlet concentrations of BF2 $(p=0.05)$ and BF4 $(p=0.047)$ for total fungi as well as between inlet and outlet samples of BF1 $(p=0.01)$ and BF3 $(p=0.021)$ for total mesophilic bacteria. For visits $13-16$, there were significant differences only between inlet and background concentrations of total fungi $(p=0.048)$ and total mesophilic bacteria $(p=0.028)$.

\section{Results and discussion}

\subsection{Operating conditions}

The operating conditions of each biofilter for the period have been summarised in table 4 . The impact of empty bed residence

Table 3

Mean bioaerosols counts and standard deviations (SD) in $\mathrm{cfu} \mathrm{m}^{-3}$.

\begin{tabular}{|c|c|c|c|c|c|c|c|c|c|c|c|c|c|c|c|c|c|c|c|c|c|c|c|c|}
\hline \multirow{3}{*}{ 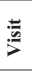 } & \multicolumn{6}{|c|}{ Aspergillus Fumigatus } & \multicolumn{6}{|c|}{ Total fungi } & \multicolumn{6}{|c|}{ Total mesophilic bacteria } & \multicolumn{6}{|c|}{ Gram negative bacteria } \\
\hline & \multicolumn{2}{|c|}{ Background* } & \multicolumn{2}{|c|}{ Inlet* } & \multicolumn{2}{|c|}{ Outlet** } & \multicolumn{2}{|c|}{ Background* } & \multicolumn{2}{|c|}{ Inlet* } & \multicolumn{2}{|c|}{ Outlet** } & \multicolumn{2}{|c|}{ Background* } & \multicolumn{2}{|c|}{ Inlet* } & \multicolumn{2}{|c|}{ Outlet** } & \multicolumn{2}{|c|}{ Background* } & \multicolumn{2}{|c|}{ Inlet* } & \multicolumn{2}{|c|}{ Outlet** } \\
\hline & Count & SD & Count & SD & Count & SD & Count & SD & Count & SD & Count & SD & Count & SD & Count & SD & Count & SD & Count & SD & Count & SD & Count & SD \\
\hline 1 & $\begin{array}{c}3.3 \times \\
10^{2}\end{array}$ & 26 & $\begin{array}{c}9.7 \times \\
10^{3}\end{array}$ & 79 & $\begin{array}{c}1.4 \times \\
10^{3}\end{array}$ & 532 & $\begin{array}{c}4.5 \times \\
10^{2}\end{array}$ & 32 & $\begin{array}{c}1.2 \times \\
10^{4}\end{array}$ & 380 & $\begin{array}{c}1.8 \times \\
10^{3}\end{array}$ & 584 & $\begin{array}{c}8.8 \times \\
10^{2}\end{array}$ & 75 & $\begin{array}{c}1.1 \times \\
10^{4}\end{array}$ & 1284 & $\begin{array}{c}3.8 \times \\
10^{3}\end{array}$ & 1317 & $\begin{array}{c}7.0 \times \\
10^{2}\end{array}$ & 46 & $\begin{array}{c}6.9 \times \\
10^{3}\end{array}$ & 1050 & $\begin{array}{c}1.3 \times \\
10^{3}\end{array}$ & 602 \\
\hline 2 & $\begin{array}{c}1.2 \times \\
10^{2}\end{array}$ & 12 & $\begin{array}{c}1.2 \times \\
10^{4}\end{array}$ & 1045 & $\begin{array}{c}2.7 \times \\
10^{3}\end{array}$ & 1032 & $\begin{array}{c}2.2 \times \\
10^{2}\end{array}$ & 10 & $\begin{array}{c}1.5 \times \\
10^{4}\end{array}$ & 1466 & $\begin{array}{c}3.2 \times \\
10^{3}\end{array}$ & 1251 & $\begin{array}{c}6.0 \times \\
10^{2}\end{array}$ & 65 & $\begin{array}{c}5.3 \times \\
10^{3}\end{array}$ & 4089 & $\begin{array}{c}8.2 \times \\
10^{3}\end{array}$ & 3260 & $\begin{array}{c}3.6 \times \\
10^{2}\end{array}$ & 55 & $\begin{array}{c}2.4 \times \\
10^{3}\end{array}$ & 188 & $\begin{array}{c}2.5 \times \\
10^{3}\end{array}$ & 620 \\
\hline 3 & $\begin{array}{c}1.5 \times \\
10^{2}\end{array}$ & 80 & $\begin{array}{c}1.0 \times \\
10^{4}\end{array}$ & 1412 & $\begin{array}{c}4.2 \times \\
10^{3}\end{array}$ & 6661 & $\begin{array}{c}1.9 \times \\
10^{2}\end{array}$ & 99 & $\begin{array}{c}1.3 \times \\
10^{4}\end{array}$ & 663 & $\begin{array}{c}4.8 \times \\
10^{3}\end{array}$ & 7464 & $\begin{array}{c}6.2 \times \\
10^{2}\end{array}$ & 18 & $\begin{array}{c}5.9 \times \\
10^{3}\end{array}$ & 76 & $\begin{array}{c}2.9 \times \\
10^{3}\end{array}$ & 1937 & $\begin{array}{c}3.9 \times \\
10^{3}\end{array}$ & 323 & $\begin{array}{c}2.5 \times \\
10^{4}\end{array}$ & 438 & $\begin{array}{c}3.6 \times \\
10^{3}\end{array}$ & 1543 \\
\hline 4 & $\begin{array}{l}1.8 \times \\
10^{2}\end{array}$ & 71 & $\begin{array}{c}7.4 \times \\
10^{3}\end{array}$ & 1981 & $\begin{array}{c}1.7 \times \\
10^{3}\end{array}$ & 1675 & $\begin{array}{c}1.9 \times \\
10^{2}\end{array}$ & 18 & $\begin{array}{c}9.3 \times \\
10^{3}\end{array}$ & 2159 & $\begin{array}{c}2.2 \times \\
10^{3}\end{array}$ & 1764 & $\begin{array}{c}7.4 \times \\
10^{2}\end{array}$ & 141 & $\begin{array}{c}2.3 \times \\
10^{4}\end{array}$ & 896 & $\begin{array}{c}4.6 \times \\
10^{3}\end{array}$ & 3467 & $\begin{array}{c}2.1 \times \\
10^{3}\end{array}$ & 281 & $\begin{array}{c}6.4 \times \\
10^{3}\end{array}$ & 131 & $\begin{array}{c}3.4 \times \\
10^{3}\end{array}$ & 945 \\
\hline 5 & $\begin{array}{c}9.8 \times \\
10^{2}\end{array}$ & 231 & $\begin{array}{c}8.1 \times \\
10^{3}\end{array}$ & 383 & $\begin{array}{c}1.0 \times \\
10^{3}\end{array}$ & 237 & $\begin{array}{c}1.1 \times \\
10^{3}\end{array}$ & 214 & $\begin{array}{c}1.0 \times \\
10^{4}\end{array}$ & 935 & $\begin{array}{c}1.6 \times \\
10^{3}\end{array}$ & 224 & $\begin{array}{c}2.6 \times \\
10^{3}\end{array}$ & 113 & $\begin{array}{c}1.3 \times \\
10^{4}\end{array}$ & 6627 & $\begin{array}{c}1.9 \times \\
10^{3}\end{array}$ & 940 & $\begin{array}{c}2.4 \times \\
10^{3}\end{array}$ & 44 & $\begin{array}{c}1.9 \times \\
10^{4}\end{array}$ & 9698 & $\begin{array}{c}6.1 \times \\
10^{3}\end{array}$ & 1912 \\
\hline 6 & $\begin{array}{c}3.0 \times \\
10^{2}\end{array}$ & 18 & $\begin{array}{c}3.8 \times \\
10^{3}\end{array}$ & 1094 & $\begin{array}{c}1.1 \times \\
10^{3}\end{array}$ & 317 & $\begin{array}{c}3.5 \times \\
10^{2}\end{array}$ & 35 & $\begin{array}{c}4.8 \times \\
10^{3}\end{array}$ & 1544 & $\begin{array}{c}1.3 \times \\
10^{3}\end{array}$ & 326 & $\begin{array}{c}1.8 \times \\
10^{4}\end{array}$ & 10226 & $\begin{array}{c}1.3 \times \\
10^{4}\end{array}$ & 832 & $\begin{array}{c}3.2 \times \\
10^{3}\end{array}$ & 1681 & $\begin{array}{c}1.5 \times \\
10^{4}\end{array}$ & 12633 & $\begin{array}{c}5.6 \times \\
10^{3}\end{array}$ & 765 & $\begin{array}{c}4.4 \times \\
10^{3}\end{array}$ & 2564 \\
\hline 7 & $\begin{array}{c}1.1 \times \\
10^{3}\end{array}$ & 398 & $\begin{array}{c}1.1 \times \\
10^{3}\end{array}$ & 286 & $\begin{array}{c}1.9 \times \\
10^{2}\end{array}$ & 116 & $\begin{array}{c}1.5 \times \\
10^{3}\end{array}$ & 800 & $\begin{array}{c}1.5 \times \\
10^{3}\end{array}$ & 413 & $\begin{array}{c}4.1 \times \\
10^{2}\end{array}$ & 306 & $\begin{array}{c}2.6 \times \\
10^{3}\end{array}$ & 396 & $\begin{array}{c}5.0 \times \\
10^{3}\end{array}$ & 2399 & $\begin{array}{c}1.8 \times \\
10^{3}\end{array}$ & 917 & $\begin{array}{c}4.8 \times \\
10^{3}\end{array}$ & 1406 & $\begin{array}{c}3.6 \times \\
10^{3}\end{array}$ & 822 & $\begin{array}{c}1.7 \times \\
10^{3}\end{array}$ & 565 \\
\hline 8 & $\begin{array}{c}6.2 \times \\
10^{2}\end{array}$ & 548 & $\begin{array}{c}1.4 \times \\
10^{3}\end{array}$ & 288 & 93 & 53 & $\begin{array}{c}6.2 \times \\
10^{2}\end{array}$ & 548 & $\begin{array}{c}1.8 \times \\
10^{3}\end{array}$ & 382 & $\begin{array}{c}1.1 \times \\
10^{2}\end{array}$ & 62 & $\begin{array}{c}1.2 \times \\
10^{3}\end{array}$ & 253 & $\begin{array}{c}9.3 \times \\
10^{3}\end{array}$ & 719 & $\begin{array}{c}1.6 \times \\
10^{3}\end{array}$ & 478 & $\begin{array}{c}3.6 \times \\
10^{3}\end{array}$ & 1979 & $\begin{array}{c}2.2 \times \\
10^{3}\end{array}$ & 565 & $\begin{array}{c}1.7 \times \\
10^{3}\end{array}$ & 732 \\
\hline 9 & $\begin{array}{c}7.1 \times \\
10^{2}\end{array}$ & 35 & $\begin{array}{c}9.6 \times \\
10^{2}\end{array}$ & 106 & 57 & 35 & $\begin{array}{c}1.1 \times \\
10^{2}\end{array}$ & 71 & $\begin{array}{c}1.2 \times \\
10^{3}\end{array}$ & 198 & 66 & 37 & $\begin{array}{c}4.0 \times \\
10^{3}\end{array}$ & 2670 & $\begin{array}{c}2.6 \times \\
10^{3}\end{array}$ & 330 & $\begin{array}{c}1.6 \times \\
10^{3}\end{array}$ & 818 & $\begin{array}{c}1.4 \times \\
10^{3}\end{array}$ & 737 & $\begin{array}{c}5.0 \times \\
10^{2}\end{array}$ & 359 & $\begin{array}{c}1.1 \times \\
10^{3}\end{array}$ & 598 \\
\hline 10 & $\begin{array}{c}7.1 \times \\
10^{2}\end{array}$ & 35 & $\begin{array}{c}6.8 \times \\
10^{2}\end{array}$ & 216 & $\begin{array}{c}6.8 \times \\
10^{2}\end{array}$ & 139 & $\begin{array}{c}7.8 \times \\
10^{2}\end{array}$ & 35 & $\begin{array}{c}9.1 \times \\
10^{2}\end{array}$ & 201 & $\begin{array}{c}7.1 \times \\
10^{2}\end{array}$ & 138 & $\begin{array}{c}1.4 \times \\
10^{3}\end{array}$ & 120 & $\begin{array}{c}6.5 \times \\
10^{2}\end{array}$ & 159 & $\begin{array}{c}1.5 \times \\
10^{3}\end{array}$ & 513 & $\begin{array}{c}1.5 \times \\
10^{3}\end{array}$ & 910 & $\begin{array}{c}1.7 \times \\
10^{3}\end{array}$ & 636 & $\begin{array}{c}1.3 \times \\
10^{3}\end{array}$ & 374 \\
\hline 11 & $\begin{array}{c}1.4 \times \\
10^{2}\end{array}$ & 35 & $\begin{array}{c}1.1 \times \\
10^{2}\end{array}$ & 106 & 44 & 66 & $\begin{array}{c}1.9 \times \\
10^{2}\end{array}$ & 18 & $\begin{array}{c}1.6 \times \\
10^{2}\end{array}$ & 124 & 80 & 82 & $\begin{array}{c}2.9 \times \\
10^{3}\end{array}$ & 443 & $\begin{array}{c}1.6 \times \\
10^{3}\end{array}$ & 30 & $\begin{array}{c}1.6 \times \\
10^{3}\end{array}$ & 735 & $\begin{array}{c}5.7 \times \\
10^{2}\end{array}$ & 143 & $\begin{array}{c}1.0 \times \\
10^{3}\end{array}$ & 323 & $\begin{array}{c}2.3 \times \\
10^{3}\end{array}$ & 1155 \\
\hline 12 & $\begin{array}{c}1.6 \times \\
10^{2}\end{array}$ & 53 & 53 & 18 & 97 & 66 & $\begin{array}{c}1.9 \times \\
10^{2}\end{array}$ & 88 & $\begin{array}{c}1.8 \times \\
10^{2}\end{array}$ & 71 & 97 & 66 & $\begin{array}{c}1.3 \times \\
10^{3}\end{array}$ & 35 & $\begin{array}{c}1.6 \times \\
10^{3}\end{array}$ & 472 & $\begin{array}{c}9.8 \times \\
10^{2}\end{array}$ & 404 & $\begin{array}{c}1.5 \times \\
10^{3}\end{array}$ & 201 & $\begin{array}{c}1.2 \times \\
10^{3}\end{array}$ & 198 & $\begin{array}{c}7.8 \times \\
10^{2}\end{array}$ & 394 \\
\hline 13 & $\begin{array}{c}9.1 \times \\
10^{2}\end{array}$ & 164 & $\begin{array}{c}1.1 \times \\
10^{4}\end{array}$ & 4488 & $\begin{array}{c}3.8 \times \\
10^{2}\end{array}$ & 481 & $\begin{array}{c}1.3 \times \\
10^{3}\end{array}$ & 147 & $\begin{array}{c}1.5 \times \\
10^{4}\end{array}$ & 6115 & $\begin{array}{c}6.5 \times \\
10^{2}\end{array}$ & 983 & $\begin{array}{c}3.7 \times \\
10^{3}\end{array}$ & 1076 & $\begin{array}{c}1.4 \times \\
10^{4}\end{array}$ & 2949 & $\begin{array}{c}2.4 \times \\
10^{3}\end{array}$ & 1120 & $\begin{array}{c}2.0 \times \\
10^{3}\end{array}$ & 528 & $\begin{array}{c}5.9 \times \\
10^{3}\end{array}$ & 1756 & $\begin{array}{c}2.1 \times \\
10^{3}\end{array}$ & 632 \\
\hline 14 & $\begin{array}{c}2.1 \times \\
10^{2}\end{array}$ & 35 & $\begin{array}{c}2.1 \times \\
10^{4}\end{array}$ & 479 & $\begin{array}{c}1.1 \times \\
10^{4}\end{array}$ & 8454 & $\begin{array}{c}7.3 \times \\
10^{2}\end{array}$ & 194 & $\begin{array}{c}4.1 \times \\
10^{4}\end{array}$ & 3827 & $\begin{array}{c}1.6 \times \\
10^{4}\end{array}$ & 12982 & $\begin{array}{c}8.2 \times \\
10^{2}\end{array}$ & 216 & $\begin{array}{c}6.0 \times \\
10^{4}\end{array}$ & 3767 & $\begin{array}{c}3.0 \times \\
10^{4}\end{array}$ & 12969 & $\begin{array}{c}1.7 \times \\
10^{3}\end{array}$ & 230 & $\begin{array}{c}1.0 \times \\
10^{4}\end{array}$ & 7943 & $\begin{array}{c}2.9 \times \\
10^{4}\end{array}$ & 17064 \\
\hline 15 & $\begin{array}{c}8.9 \times \\
10^{2}\end{array}$ & 37 & $\begin{array}{c}1.0 \times \\
10^{4}\end{array}$ & 1988 & $\begin{array}{c}4.2 \times \\
10^{3}\end{array}$ & 1090 & $\begin{array}{c}1.1 \times \\
10^{3}\end{array}$ & 94 & $\begin{array}{c}1.2 \times \\
10^{4}\end{array}$ & 2072 & $\begin{array}{c}5.7 \times \\
10^{3}\end{array}$ & 1408 & $\begin{array}{c}6.9 \times \\
10^{2}\end{array}$ & 53 & $\begin{array}{c}4.7 \times \\
10^{4}\end{array}$ & 1276 & $\begin{array}{c}2.2 \times \\
10^{4}\end{array}$ & 10116 & $\begin{array}{c}1.2 \times \\
10^{3}\end{array}$ & 104 & $\begin{array}{c}2.6 \times \\
10^{4}\end{array}$ & 14846 & $\begin{array}{c}2.1 \times \\
10^{4}\end{array}$ & 13616 \\
\hline 16 & $\begin{array}{c}6.0 \times \\
10^{2}\end{array}$ & 35 & $\begin{array}{c}5.3 \times \\
10^{4}\end{array}$ & 9154 & $\begin{array}{c}3.4 \times \\
10^{4}\end{array}$ & 4122 & $\begin{array}{c}8.9 \times \\
10^{2}\end{array}$ & 111 & $\begin{array}{c}6.3 \times \\
10^{4}\end{array}$ & 11843 & $\begin{array}{c}4.0 \times \\
10^{4}\end{array}$ & 5448 & $\begin{array}{c}5.2 \times \\
10^{3}\end{array}$ & 300 & $\begin{array}{c}9.6 \times \\
10^{4}\end{array}$ & 25016 & $\begin{array}{c}5.1 \times \\
10^{4}\end{array}$ & 22975 & $\begin{array}{c}2.3 \times \\
10^{3}\end{array}$ & 945 & $\begin{array}{c}2.8 \times \\
10^{4}\end{array}$ & 4620 & $\begin{array}{c}1.3 \times \\
10^{4}\end{array}$ & 5011 \\
\hline
\end{tabular}

SD: Standard Deviation; ${ }^{*} \mathrm{n}=2 ;{ }^{* *} \mathrm{n}=8$; shaded rows $=$ outdoor sampling 
Table 4

Operating conditions of the biofilters (BF) when operated within (visits 1-6, 13-16) and outside (visits 7-12) the building.

\begin{tabular}{|c|c|c|c|c|}
\hline \multicolumn{2}{|c|}{ Parameter } & \multicolumn{3}{|l|}{ Visits } \\
\hline & & \multirow{2}{*}{$\begin{array}{l}1-6 \\
23.8\end{array}$} & $7-12$ & $13-16$ \\
\hline \multicolumn{2}{|c|}{ Mean inlet air temperature $\left({ }^{\circ} \mathrm{C}\right)$} & & 15.8 & 15.2 \\
\hline \multirow[t]{5}{*}{ BF1 } & Leachate $\mathrm{pH}$ range & $5.19-6.52$ & $6.52-7.0$ & $6.83-7.04$ \\
\hline & Mean EBRT (s) & 16,70 & 16 & 16 \\
\hline & Mean Airflow rate $\left(\mathrm{L} \mathrm{min}^{-1}\right)$ & 681,156 & 681 & 681 \\
\hline & Mean media temperature $\left({ }^{\circ} \mathrm{C}\right)$ & 19.5 & 15.1 & 13.8 \\
\hline & Mean outlet air temperature $\left({ }^{\circ} \mathrm{C}\right)$ & 21.8 & 13.1 & 14.5 \\
\hline \multirow[t]{5}{*}{ BF2 } & Leachate $\mathrm{pH}$ range & $5.12-6.64$ & $6.62-7.52$ & $6.56-7.38$ \\
\hline & Mean EBRT (s) & 16,11 & 16 & 16 \\
\hline & Mean Airflow rate $\left(\mathrm{L} \mathrm{min}^{-1}\right)$ & 681,990 & 681 & 681 \\
\hline & Mean media temperature $\left({ }^{\circ} \mathrm{C}\right)$ & 20.3 & 13.9 & 13.6 \\
\hline & Mean outlet air temperature $\left({ }^{\circ} \mathrm{C}\right)$ & 21.4 & 13.8 & 14.5 \\
\hline \multirow[t]{5}{*}{ BF3 } & Leachate $\mathrm{pH}$ range & $6.17-7.04$ & $6.98-7.34$ & $6.77-7.37$ \\
\hline & Mean EBRT (s) & 16,11 & 16 & 16 \\
\hline & Mean Airflow rate $\left(\mathrm{L} \mathrm{min}^{-1}\right)$ & 681,990 & 681 & 681 \\
\hline & Mean media temperature $\left({ }^{\circ} \mathrm{C}\right)$ & 21.2 & 14.5 & 15.3 \\
\hline & Mean outlet air temperature $\left({ }^{\circ} \mathrm{C}\right)$ & 21.4 & 13.0 & 14.6 \\
\hline \multirow[t]{5}{*}{ BF4 } & Leachate $\mathrm{pH}$ range & $5.55-6.53$ & $6.43-7.44$ & $6.95-7.21$ \\
\hline & Mean EBRT (s) & 16,70 & 16 & 16 \\
\hline & Mean Airflow rate $\left(\mathrm{L} \mathrm{min}^{-1}\right)$ & 681,156 & 681 & 681 \\
\hline & Mean media temperature $\left({ }^{\circ} \mathrm{C}\right)$ & 21.2 & 14.6 & 16.1 \\
\hline & Mean outlet air temperature $\left({ }^{\circ} \mathrm{C}\right)$ & 21.1 & 13.5 & 14.3 \\
\hline
\end{tabular}

time was assessed during the first six sampling visits by comparing the outlet bioaoerosol concentration for the four biofilters. During the first three sampling visits the biofilters were all adjusted to run at an average empty bed residence time of $16 \mathrm{~s}$. An assessment of the outlet bioaerosol concentrations showed that there were no outliers and the data was normally distributed for each group as assessed by Shapiro-Wilk test $(p<.05)$. However, there was heterogeneity of variances for A. fumigatus $(p=.003)$ and total fungi $(p=.004)$ as assessed by Levene's test of homogeneity of variance; there was no statistically significant differences in the outlet concentrations of A. fumigatus $(p=.433)$ and total fungi $(p=.482)$ from all four biofilters as assessed with Welch ANOVA. One way ANOVA also indicated that there was no statistically significant difference in the outlet concentrations for total bacteria $(p=.670)$ and Gram negative bacteria $(p=.594)$.

For visits 4-6, BF1 and BF4 were randomly selected to operate at an average EBRT of $70 \mathrm{~s}$ while BF2 and BF3 had an average EBRT of $11 \mathrm{~s}$. This was done to assess whether there were contact time dependent significant differences in the measured outlet bioaerosol concentrations between the two groups of biofilters. Welch ANOVA indicated that there was no statistically significant difference between all outlet concentrations for A. fumigatus ( $p=.407$ ), total fungi $(p=.425)$ and total bacteria $(p=.243)$. For Gram negative bacteria, one way ANOVA also showed no statistically significant difference $(p=.148)$ in the outlets from the four biofilters. In summary, there was no significant difference in the performance of the biofilters when operated under varying conditions of EBRT. Limited statistical power due to the modest sample size in this study ( $n=64)$ may have played a role in limiting the significance of some of the statistical comparisons carried out (Cornish, 2006). Post hoc power analysis, with power $(1-\beta)$ set at 0.90 and $\alpha=0.05$, indicated that sample size would have to increase up to 95 samples for group differences to reach statistical significance at the 0.05 level.

Odour control function of biofilters is dependent on the activity of microbial population within the media. These microorganisms thrive at $\mathrm{pH}$ range of $6.5-8$ which must be maintained within the internal environment of the biofilter (Wani et al., 1997; Schnelle and Brown, 2002). However, to evaluate the performance for bioaerosol control, the biofilters were operated without any supplementary attempts to alter the $\mathrm{pH}$ which was in the range of 5.12-7.52 for all four biofilters. Also, no adjustments were made to alter the media temperature especially as these were within the optimal levels $\left(10-40^{\circ} \mathrm{C}\right)$ recommended for biological treatment systems (Schnelle and Brown, 2002).

\subsection{Removal efficiency}

The first set of results considers the removal efficiency of the biofilters under conditions of high inlet bioaerosol concentrations under summer (visits 1-6) and winter (visits 13-16) conditions. Fig. 4 shows the RE and concentrations of each group of bioaerosols sampled at the different sampling points (background, biofilter inlet and outlets) plotted against the site visits conducted for this study. There was no significant difference between the performances of the four pilot-scale biofilters (Section 3.1), hence the REs were computed using the mean outlet concentrations from the four reactors for each visit.

During visits $1-6$, inlet $A$. fumigatus concentration ranged from $3.8 \times 10^{3}$ to $1.2 \times 10^{4} \mathrm{cfu} \mathrm{m}^{-3}$ for which the biofilters achieved RE of $60-88 \%$, giving outlet concentrations between $1.0 \times 10^{3}$ to $4.2 \times 10^{3} \mathrm{cfu} \mathrm{m}^{-3}$. Similarly, the biofilters achieved RE of $65-85 \%$ for total fungi with inlet concentration in the range of $4.8 \times 10^{3}$ to $1.5 \times 10^{4} \mathrm{cfu} \mathrm{m}^{-3}$ and delivering outlet concentrations between $1.3 \times 10^{3}$ to $4.8 \times 10^{4} \mathrm{cfu} \mathrm{m}^{-3}$. A. fumigatus particles constituted approximately $80 \%$ of the total fungi particles, comparable to the study of Millner et al. (1977) who reported that A. fumigatus made up $75 \%$ of the total viable mycoflora captured on the compost site studied. For this same period, slightly lower RE of $52-86 \%$ was recorded for the total mesophilic bacteria with outlet concentration of $1.9 \times 10^{3}$ to $8.2 \times 10^{3} \mathrm{cfu} \mathrm{m}^{-3}$ from inlet concentration $5.9 \times 10^{3}$ to $5.3 \times 10^{4} \mathrm{cfu} \mathrm{m}^{-3}$ while the biofilter achieved a much lower RE of -4.1 to $86 \%$ for Gram negative bacteria, treating inlet concentration between $2.4 \times 10^{3}$ to $2.5 \times 10^{4} \mathrm{cfu} \mathrm{m}^{-3}$. The data suggest that variation of empty bed residence time (between $11 \mathrm{~s}, 16 \mathrm{~s}$ and $70 \mathrm{~s}$ for this study) did not influence RE for the four groups of bioaerosols measured. This observation is supported by data presented by Sanchez-Monedero et al. (2003) which showed that RE for A. fumigatus did not appear to be related to the gas phase residence times of biofilters which operated in the range of 29-97 s, and achieved RE $>90 \%$. Similarly, no relationship was found between gas phase residence time and the RE for mesophilic 


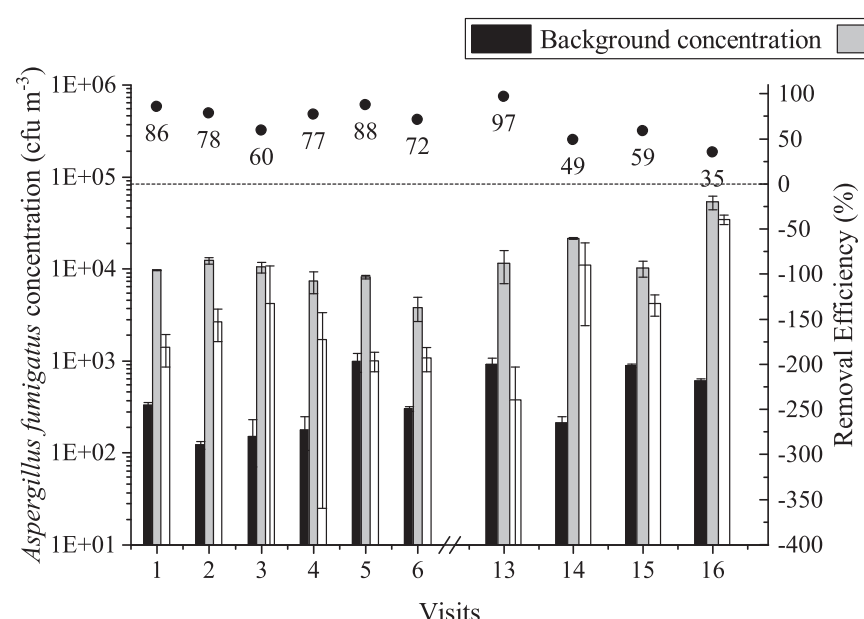

(a)

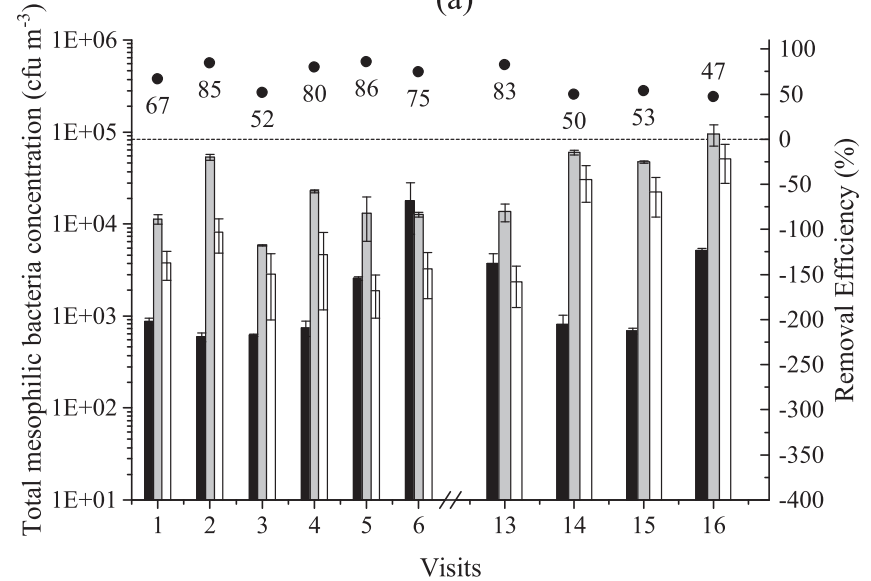

(c)

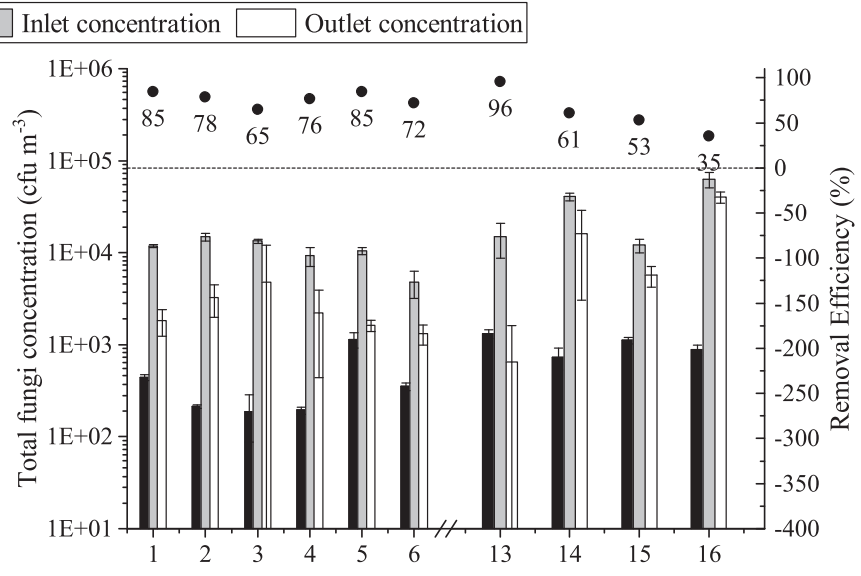

(b)

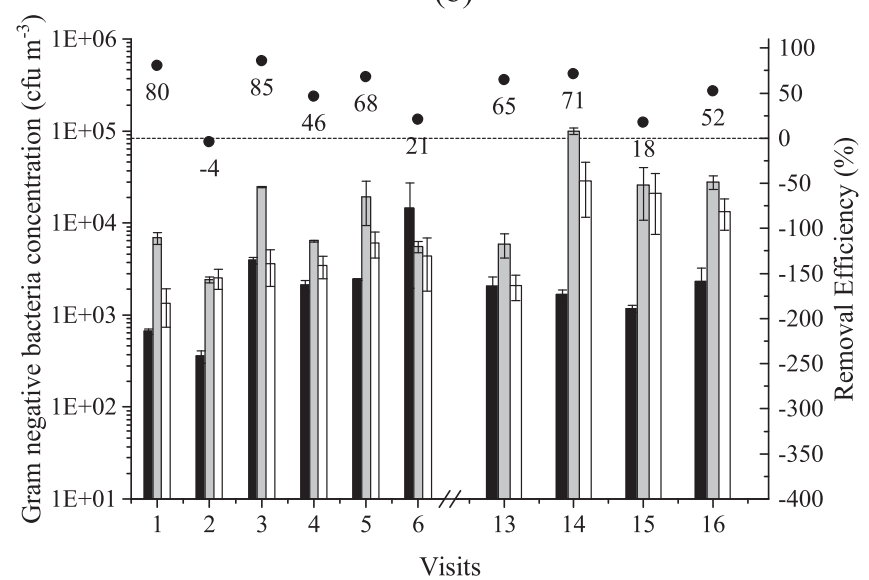

(d)

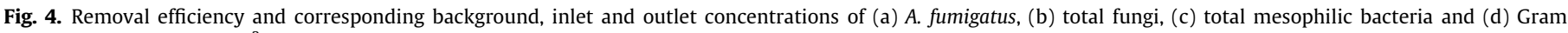
negative bacteria in $\mathrm{cfu} \mathrm{m}^{-3}$ when biofilters were operated within the building. (Error bars = standard deviation).

bacteria (highest: $89.6 \%$ at 36 s, and lowest: $39.1 \%$ at 37 s), suggesting that gas phase residence time may not play a significant role in the capture of aerosolised bacteria and fungi.

Leson and Winer (1991) recommended typical residence times of 25-60 s for commercial or industrial biofilter applications for odour and low volatile organic compound (VOC) abatement, and gas phase residence times less than $23 \mathrm{~s}$ have been shown to cause resistance of the transfer of hydrogen sulphide from the gas phase into the biofilm layer of the media (Yang and Allen, 1994). It therefore suggests that significant bioaerosol RE is achievable across a range of EBRTs that can deliver both poor and optimum odour control. Martens et al. (2001) in their research suggested that bioflters which were excellent odour abatement systems emitted slightly more bioaerosols particles. However, they could not establish any relationships between the removal efficiencies of the odour/ ammonia and bioaerosols for the five filter materials (i.e. biochips, coconut-peat, wood-bark, pellets \& bark, and compost) tested. Bioaerosol capture mechanisms include inertial deposition, diffusional or Brownian deposition and flow line interception (Ottengraf and Konings, 1991); and these combine to effect bioaerosol impingement on the solid media material such that as bioaerosol-laden air sweeps through the media bed, the particles get deposited within the media, a function which may not be dependent on gas contact time. This further suggests that a low EBRT biofilter which may not favour odour control may actually achieve significant bioaerosol control. However, this observation may have been influenced by the small sample size and the variability in the dataset, and so valid conclusions would require an extensive study with a larger sample size. Nonetheless, Fletcher et al. (2014) argued that it may not be possible to achieve simultaneous significant control of odour and bioaerosols within a single biofilter as the mechanisms involved in the removal of these two pollutants are different. They also noted that bioaerosol removal may be enhanced by increasing airflow which decreases the EBRT.

In winter conditions (visits 13-16), the sampling yielded REs of $60 \%$ (35-97\%), 61\% (35-96\%), 58\% (47-83\%) and 51\% (18-71\%) for A. fumigatus, total fungi, total mesophilic bacteria and Gram negative bacteria, respectively. It was observed that the inlet concentrations during visits 13-16 (winter) were significantly higher $(p<.05)$ than during visits $1-6$ (summer), up to $5.3 \times 10^{4} \mathrm{cfu} \mathrm{m}^{-3}$, $6.3 \times 10^{4} \mathrm{cfu} \mathrm{m}^{-3}, 9.6 \times 10^{4} \mathrm{cfu} \mathrm{m}^{-3}$ and $1.0 \times 10^{5} \mathrm{cfu} \mathrm{m}^{-3}$ for $A$. fumigatus, total fungi, total mesophilic bacteria and Gram negative bacteria, respectively. It is unclear why this was so, especially as bioaerosols concentrations tend to be higher in summer for most waste management facilities (Stagg et al., 2010). However, it was observed that the volume of waste heap in the waste reception area were greater in the winter than in summer, thus, there were increased activity of the front loaders and dinosaurus machine to feed the conveyors while clearing the area for incoming loads. Searl (2008) noted that bioaerosol concentrations can fluctuate over short periods, and increased activity levels within the waste facility may be associated with higher bioaerosol exposure. Thus, the higher winter concentrations in this study could be a function 
of the increased activities due to huge volume of waste being processed. Furthermore, Nasir and Tyrrel (2017) concluded that bioaerosol emissions from waste treatment facilities can be highly variable and characterisation based on snapshot and infrequent sampling may not give a true reflection of the magnitude of emissions. Most waste management facilities have as part of their permit condition the need to demonstrate that they can meet required emissions limit values. In this study, in spite of the high REs achieved during summer and winter $(>80 \%)$, the measured outlet concentrations still exceed background (upward) concentration, and are often in excess of the guideline provided in the EA position statement and so might be of concern to site workers and members of public living in the vicinity of site if these were operated at full scale.

\subsection{Potential for emissions from biofilters}

As earlier noted, one of the key concerns with biofilters has been their potential to act as net emitters of bioaerosols - this being one of the major concerns for regulators and operators (Fletcher et al., 2014). During sampling visits 7-12, the biofiltration system treated relatively less polluted air with inlet concentrations in the range of 53 to $1.4 \times 10^{3} \mathrm{cfu} \mathrm{m}^{-3}, 1.6 \times 10^{2}$ to $1.8 \times 10^{3}$ cfu m $\mathrm{m}^{-3}, 6.5 \times 10^{2}$ to $9.3 \times 10^{3} \mathrm{cfu} \mathrm{m}^{-3}$ and $5.0 \times 10^{2}$ to $3.6 \times 10^{3}-$ cfu $\mathrm{m}^{-3}$ for A. fumigatus, total fungi, total mesophilic bacteria and Gram negative bacteria, respectively. The results in Fig. 5 show that REs drop significantly and in some cases become negative with values as low as $-83 \%$ ( . fumigatus), $-122 \%$ (total mesophilic bacteria) and $-128 \%$ (Gram negative bacteria). The negative removal efficiencies are indicative of a greater concentration leaving the biofilter than entering and are thought to result from microorganisms (a) passing through the media, and/or (b) growing within the media and released from it (Sanchez-Monedero et al., 2003). Fletcher stated that approximately $10^{7}$ microorganisms/g colonise media surfaces; and some of these could become mobilised as air passes through the biofilter and so may result in higher concentration of bioaersols in treated air than in untreated air (Rabe and Becker, 2000). Martens et al. (2001) also added that this may still occur even if the packing material can somewhat contain the bioaerosol in contaminated; thus, suggesting some contribution to the emitted bioaerosol concentration from the media microorganisms. Laboratory scale studies by Frederickson et al. (2013) also suggested that woodchips and peat based biofilters could be net emitters of total mesophilic bacteria and gram-negative bacteria.

\subsection{Relationship between RE and inlet concentration}

The relationship between the $\log 10$ of inlet bioaerosol concentration and the removal efficiency was investigated through a linear regression analysis using data from all visits (Fig. 6). A statistically significant relationship was found for total mesophilic bacteria and Gram negative bacteria where, $p<.0005$ was found for both intercept and slope coefficient; $\log 10$ of inlet concentration accounted for $35.5 \%$ and $37.0 \%$ of the explained variability in

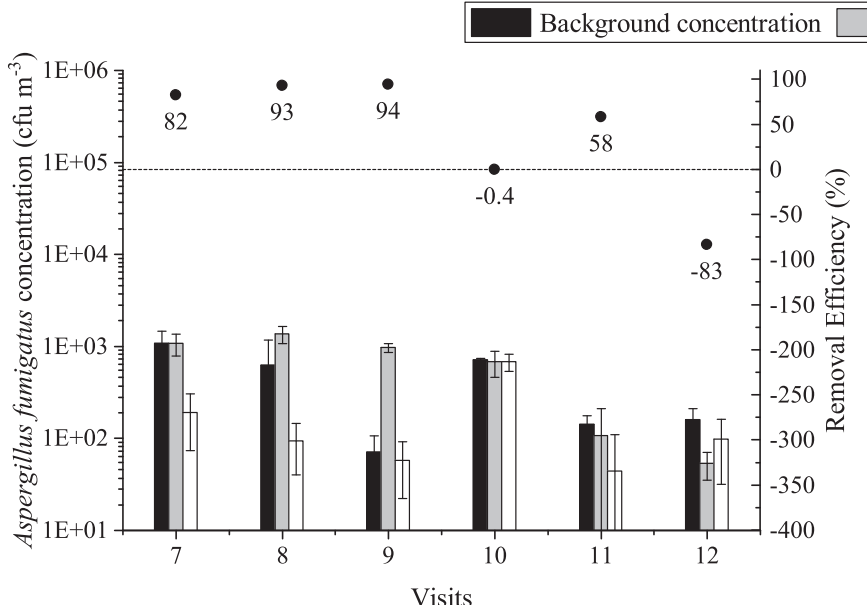

(a)

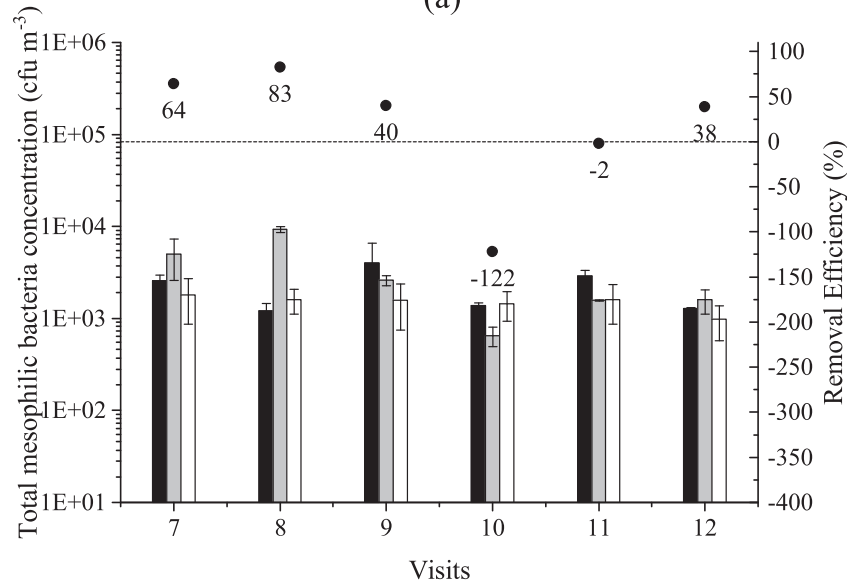

(c)

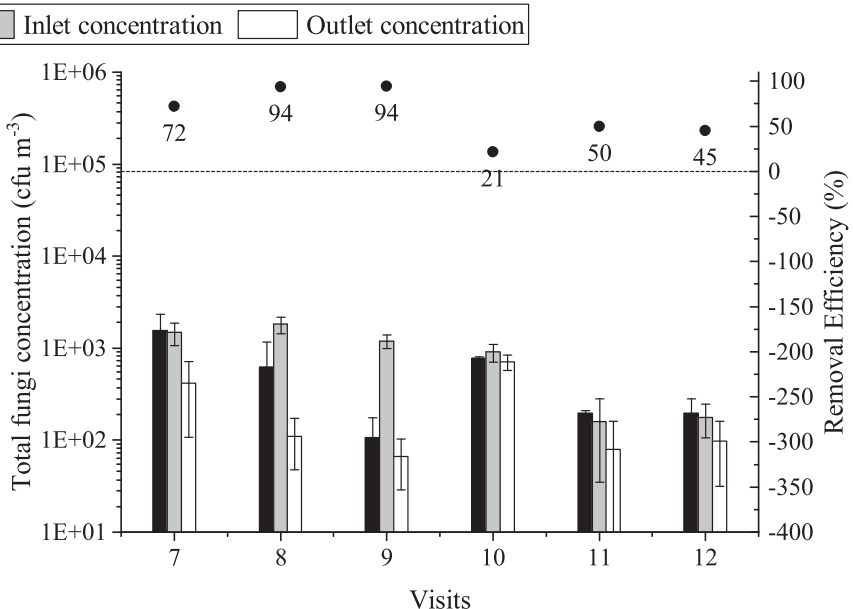

(b)

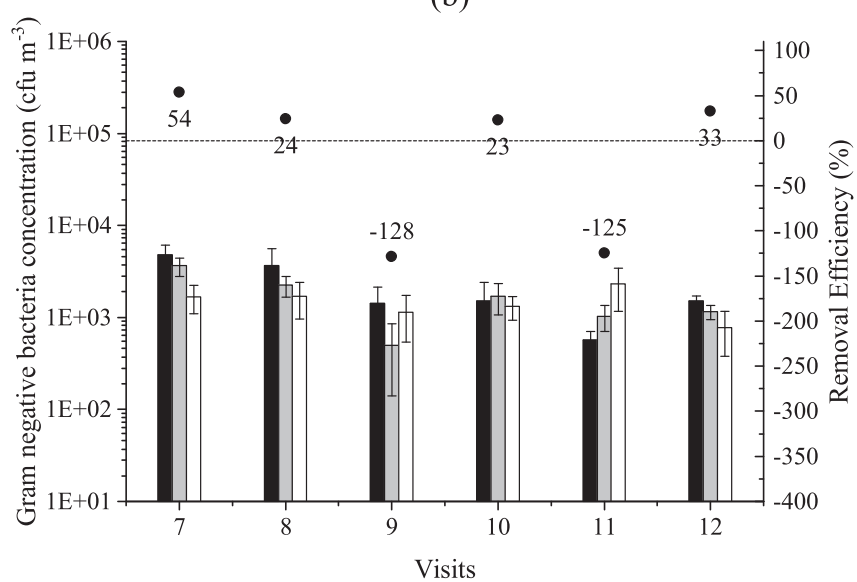

(d)

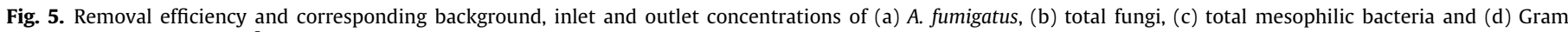
negative bacteria in $\mathrm{cfu} \mathrm{m}^{-3}$ when biofilters were operated outside the building. (Error bars = standard deviation). 


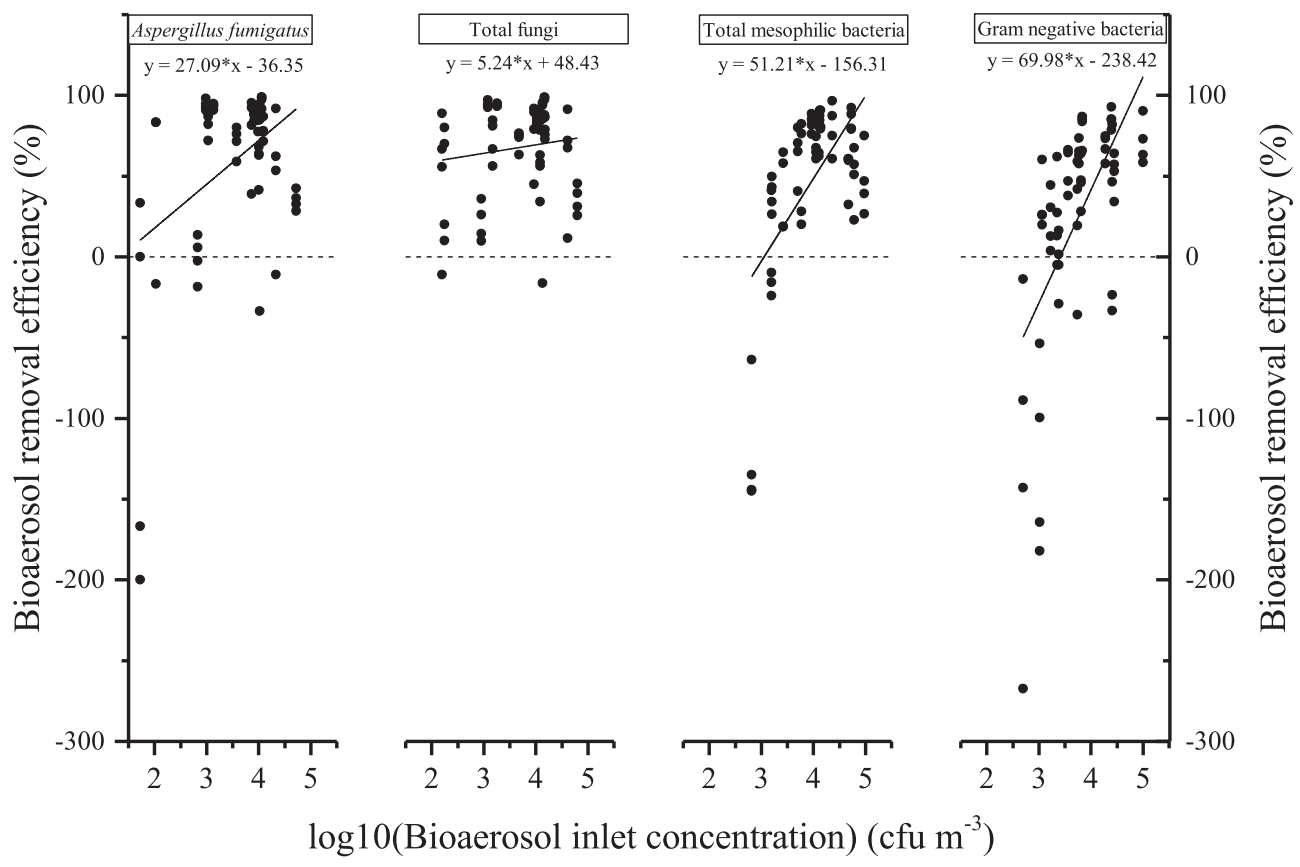

Fig. 6. Linear regression between log of inlet bioaerosol concentration and removal efficiency.

the RE for total mesophilic bacteria and Gram negative bacteria, respectively. On the other hand, a statistical relationship could not be obtained for A. fumigatus (intercept [ $p=.213$ ]; slope coefficient $[p<.0005]$ ) and total fungi (intercept $[p<.0005]$; slope coefficient $[p=.290]$ ) where log 10 of inlet concentration accounted for only $15.6 \%$ and $1.8 \%$ of the explained variability for A. fumigatus and total fungi, respectively. This indicates a much better reliability of the regression model for total mesophilic bacteria and Gram negative bacteria removal when compared to A. fumigatus and total fungi. This also suggests that differences exist between the ability of the biofiltration system to deal with fungi and bacteria, and these may be related to particle size (Sanchez-Monedero et al., 2003; Frederickson et al., 2013). Fig. 6 also shows a higher variability in performance at low inlet concentration than at high inlet concentration especially for A. fumigatus, total mesophilic and Gram negative bacteria. This may be that biofilters receiving low inlet concentrations perform more poorly than when they receive waste gas with high inlet concentrations. However, it may be that there is always a small emission rate from a biofilter, but this only becomes apparent when the inlet concentration is low; when inlet concentrations are high the removal may be the dominant process, with any emissions masked by this high removal rate. Martens et al. (2001), in their study on biofiltration of a pig facility, explained that microbial loads emitted from biofilters are a summation of non-impacted microorganisms retained in the treated process air and those blown off from the surface of the media particles by the passing airstream, thus, suggesting the possibility of that the species composition of the outlet air may be different from those of the inlet even for this study. Nonetheless, this is a promising result since the reality for most facilities would be high inlet concentrations, unless they have an upstream scrubber which reduces the concentration in the air before entering the biofilter bed (Fletcher et al., 2014).

\subsection{Size distribution of bioaerosols}

To further evaluate the potential impact of the outlet air in a real life scenario, it was imperative to assess size distribution of bioaerosols in biofilter exhausted air and to relate these to the tidal volume inhaled by humans. Particles collected on the various stages of the Andersen sampler represent a profile of their lung penetration, and so is indicative of the location of their deposition in the human respiratory tract (Andersen Instruments, 1984). Stages 1 and 2 of the sampler collect particles with aerodynamic diameter $>4.7 \mu \mathrm{m}$, which equates to nasal deposition, stages 3 and 4 collects particles with aerodynamic diameter 2.1 to $4.7 \mu \mathrm{m}$, which equates to bronchial deposition, and stages 5 and 6 collects particles $<2.1 \mu \mathrm{m}$, which equates to alveolar deposition. These correspond to the inhalable, thoracic and respirable fractions, respectively, described in TSI Incorporated (2013).

Fig. 7 shows the variation in size distribution of bioaerosol particles collected at the different stages of the six-stage Andersen sampler. The size distribution was computed by taking into account all the samples taken during sampling visits 1-6 and 13-16 (when the biofilters were located indoors) from the background, inlet and all four biofilters' outlets. More than $60 \%$ of background A. fumigatus and total fungi particles were sized $>2.1 \mu \mathrm{m}$ in aerodynamic diameter. On the other hand, background Gram negative bacteria had $\sim 50 \%$ of particles in this range, but when considering total mesophilic bacteria, the proportion of particles in this range was slightly $<50 \%$ of a concentration of $6.2 \times 10^{2}$ to $1.8 \times 10^{4} \mathrm{cfu} \mathrm{m}^{-3}$. All four biofilter outlets had $\sim 40 \%$ of A. fumigatus (outlet concentration: $3.8 \times 10^{2}$ to $3.4 \times 10^{4} \mathrm{cfu} \mathrm{m}^{-3}$ ) and total fungi (outlet concentration: $6.5 \times 10^{2}$ to $4.0 \times 10^{4} \mathrm{cfu} \mathrm{m}^{-3}$ ) particles with aerodynamic diameter $<2.1 \mu \mathrm{m}$, similar to their background composition. This is in contrast to the inlet samples that had $\sim 20 \%$ of $A$. fumigatus particles (inlet concentration range: $3.8 \times 10^{3}$ to $5.3 \times 10^{4} \mathrm{cfu} \mathrm{m}^{-3}$ ) and total fungi (inlet concentration range: $4.8 \times 10^{3}$ to $6.3 \times 10^{4} \mathrm{cfu} \mathrm{m}^{-3}$ ) particles $<2.1 \mu \mathrm{m}$, respectively. For total mesophilic bacteria (with inlet concentration range of $5.9 \times 10^{3}$ to $9.6 \times 10^{4} \mathrm{cfu} \mathrm{m}^{-3}$ ), the inlet samples had $\sim 50 \%$ particles $<2.1 \mu \mathrm{m}$ while the outlet samples were composed of $\sim 70 \%$ of particles in this range (outlet concentration $1.9 \times 10^{3}$ to $5.1 \times 10^{4}$ $\mathrm{cfu} \mathrm{m}^{-3}$ ). Inlet and outlets particle size distributions for Gram negative bacteria were comparable with $\sim 60 \%$ of particles $<2.1 \mu \mathrm{m}$, except for biofilter 4 that was slightly less than $60 \%$.

Overall, the exhausted (outlet) air appears to have smaller particles than the air entering the system even with significantly high 


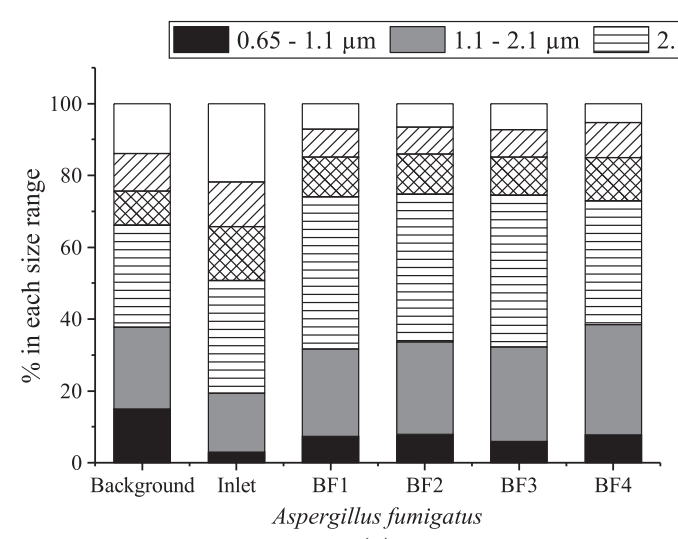

(a)

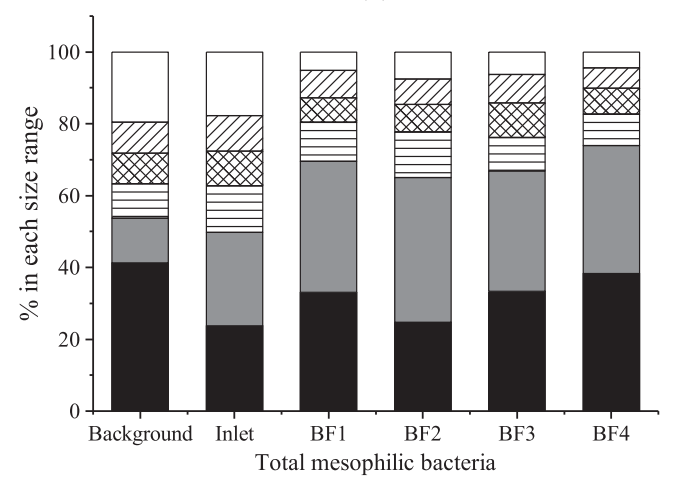

(c)

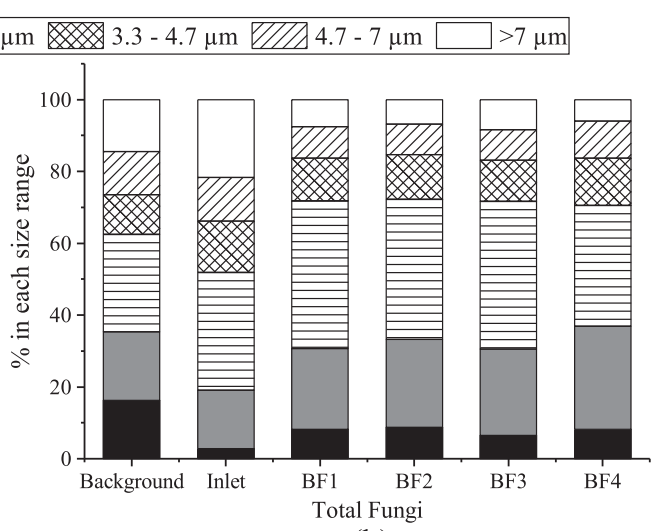

(b)

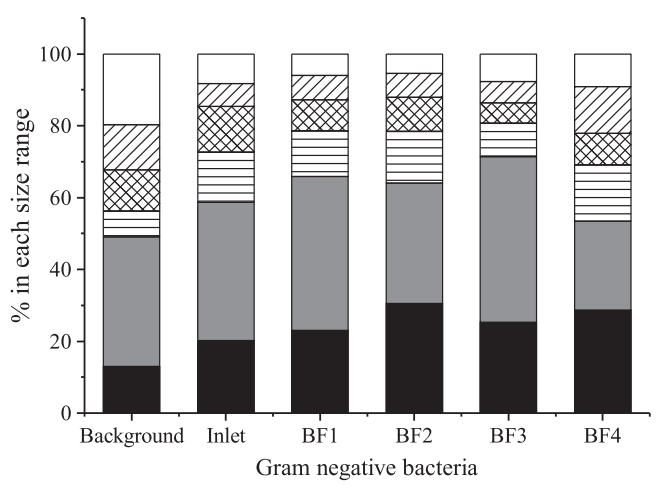

(d)

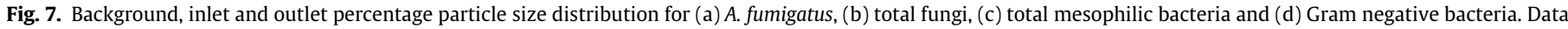
based on the ten indoor sampling visits 1-6, 13-16. Outlet composition represented by BF1, BF2, BF3, BF4.

REs recorded when the biofiltration system was operated indoors. This could possibly result from the filter bed preferentially trapping the larger sized particles from the gas flow, and/or these may just be the size range emitted from the biofilters (SanchezMonedero et al., 2003). However, as these pilot-scale biofilters achieved outlet concentrations predominantly in the range of $10^{2}-10^{3} \mathrm{cfu} \mathrm{m}^{-3}$, these concentrations would further be reduced (by wind dilution) downwind in full-scale applications. Williams et al. (2013), in a study to provide evidence on bioaerosol production, dispersion and potential exposures from four different composting facilities within England, reported peak total bacteria concentrations of $>10^{6} \mathrm{cfu} \mathrm{m}^{-3}$ immediately downwind of the sites in comparisons to the $<10^{3} \mathrm{cfu} \mathrm{m}^{-3}$ recorded upwind. However, the concentrations were noticed to decline at locations further downwind of the sites which is in agreement with the view that bioaerosol concentrations levels tend to reach background levels within $250 \mathrm{~m}$ of their point of origin (Pankhurst et al., 2011). Nonetheless, it may also not be possible to make this generalisation especially as these concentrations were measured at pilot scale within the waste hall, and so impact of fugitive emissions and other outdoor sources (Taha et al., 2004; Parry, 2018) were not assessed downwind of site and/or close to sensitive receptors.

For all sampling points, both $A$. fumigatus and total fungi showed a maximum particle size distribution at stage 4 corresponding to an average aerodynamic diameter between 2.1 and $3.3 \mu \mathrm{m}$, according the specification of the sampler. Total mesophilic bacteria and Gram negative bacteria size distribution both showed a maximum at stage 5 corresponding to an average aerodynamic diameter between 1.1 and $2.1 \mu \mathrm{m}$. These observations are in comparison to those of Sanchez-Monedero et al. (2003) who stated that this would imply a much better RE for the fungal spores; however, that size difference alone would not be sufficient to explain the observed difference in REs of fungi and bacteria measured in their study as well as in this study. Particle shape has also been suggested as having a key influence on particle retention (Willeke et al., 1996); and for particles with aerodynamic diameter $<1 \mu \mathrm{m}$, Sanchez-Monedero et al. (2003) suggested that electrostatic charge on the particles may also influence particle deposition on the biofilter media.

Bioaerosol particle size plays a key role in their dispersion in air and subsequent potential risk upon exposure via inhalation (Ferguson et al., 2017). Kell et al. (1998) argued that the potential for harmful effects by bioaerosols, upon deposition, is dependent on the number of culturable organisms, and not the culturable particles. Ferguson et al. (2017) reported that bacterial community structure and abundance were size related. They argued that since viable bacterial bioaerosols could exist either as single cells, small aggregates of cells or conglomerates of cells, then bacterial bioaerosols in stages $<3.3 \mu \mathrm{m}$ were single cells while those in stages $>3.3 \mu \mathrm{m}$ were either conglomerates of bacterial cells or cells attached to larger particles e.g. water droplets or dust. This latter group also showed more abundance and diversity with the highest levels found in the largest $(>7 \mu \mathrm{m})$ size class. Thus, with predominantly lower size class in the outlet air, the potential to cause ill health from exposure would be determined by a knowledge of the species composition of the samples which was beyond the scope of this study.

Comparison of maximum outlet to inlet respirable fractions of bioaerosols shows a ratio of 1:0.8 for both A. fumigatus and total fungi. This implies that more fungi particles in this size class were released from the biofilters than received with outlet concentrations of $1.4 \times 10^{4} \mathrm{cfu} \mathrm{m}^{-3}$ and $1.6 \times 10^{4} \mathrm{cfu} \mathrm{m}^{-3}$ for $A$. fumigatus and total fungi, respectively. On the contrary, total mesophilic bacteria showed a 1:1.3 ratio of outlet to inlet respirable particles 
while Gram negative bacteria had a 1:3.4 outlet to inlet respirable faction ratio. This indicates that the biofilters were better at controlling this fraction of bacterial particles which represent a greater human health risk as they can penetrate the respiratory system more deeply and even to the lung alveoli where gaseous exchange occurs.

Currently, there are no occupational exposure limits for bioaerosols in the UK; comparisons are usually made with other studies and publications on typical concentrations for similar facilities (Stagg et al., 2013). Malmros et al. (1992) suggested that waste workers should not be exposed to concentrations of total bacteria exceeding 5000 to $10000 \mathrm{cfu} \mathrm{m}^{-3}$ for an $8 \mathrm{~h}$ working period; thus, the concentrations reported for this facility present potential health risks to the workers on this site. The study by Stagg et al. (2013) on seven materials recycling facility within the UK indicated similar concentrations observed in this study, and at those concentrations several health problems were triggered including skin symptoms, respiratory symptoms, and gastrointestinal symptoms. However, workers' health impact assessment was outside the scope of this study. Nonetheless, the respiratory-related symptoms observed in the study by Stagg et al. (2013) can be a function of the lung penetrability of the bioaerosol particles generated at the various operational activities within the waste hall. This study indicates that approximately $20 \%, 20 \%, 50 \%$ and $60 \%$ of indoor concentrations of $A$. fumigatus, total fungi, total mesophilic bacteria and Gram negative bacteria, respectively, were respirable fractions (with aerodynamic diameter $<2.1 \mu \mathrm{m}$ ), and so could penetrate deep into the lungs.

Tidal volume, which is the volume of air inspired or expired during a respiratory cycle (Quanjer et al., 1993), is approximately $500 \mathrm{ml}$ and at rest a normal human being has 12 breaths per minute (Meka and Van Oostrom, 2004). For an 8-hour working period a normal person working continuously in the vicinity of the biofilters may beinhaling $2.88 \mathrm{~m}^{3}$ of air containing approximately $3.9 \times 10^{4} \mathrm{cfu}$ of $A$. fumigatus, $4.6 \times 10^{4} \mathrm{cfu}$ of total fungi, $1.0 \times 10^{5} \mathrm{cfu}$ of total mesophilic bacteria and $5.0 \times 10^{4} \mathrm{cfu}$ of Gram negative bacteria respirable fractions. However, these values represent the maximum concentrations recorded during this study, and do not typify the outlet concentration ranges. Nonetheless, it is estimated that $<10 \%$ of all bioaerosols may be culturable (Blomquist, 1994; Swan et al., 2003), the remainder possibly being composed of either viable non-culturable cells or dead but intact cells which may still pose health concerns (Pearson et al., 2015). Thus, the reality might be that the actual bioaerosol concentration emitted by the biofilters may be higher than measured, and may contain species or cell components that are not detected, which still require consideration in health impact assessment (Eduard et al., 2012). Even with the measured outlet concentrations, it is expected that further reduction by microbial inactivation due to environmental stresses (such as desiccation, temperature and oxygen) (Hurst et al., 2007), and wind dilution and dispersion (as they are blown off the site) would be achieved in full-scale applications.

\subsection{Applicability of results}

The application of a pilot scale biofilter in this study has provided new insights into bioaerosols removal including relationships between operating parameters, removal efficiency, size distribution of microorganism in air and potential for emissions. The application of the pilot scale biofilter within an MRF facility provided an opportunity to collect data that is closer to realworld biofilter operation than a highly controlled laboratory study. However it should be noted that this brings with it some uncertainties and limitations and hence the results of this study may not allow for generalisation of conclusions for various reasons.

Although biofilters have been applied to MBT plants (Stagg et al., 2013), they are less common in MRF plants. These facilities are generally fairly clean and do not have the levels of organic dust and odour found in facilities such as in-vessel composting (IVC) (Surrey County Council, 2017) where biofilters are likely to be better suited. The location of the pilot system (away from the waste heap behind the back-push wall) is another factor which could have impacted on the results especially as this area was relatively cleaner than other areas within the waste hall. For these reasons, the measured bioaerosol concentrations have to be considered relative to those levels typical in facilities that generate much higher concentrations, and it is not clear whether the same findings would be apparent under much higher bioaerosols loads. Nonetheless, this study was based in this MRF to enable the evaluation of the system with the real source of bioaerosols associated with this type of waste being processed, and hence the findings are likely to be applicable to other comparable MRF facilities.

Secondly, the sampling methods employed in this study were targeted at assessing total bioaerosol loads removal by biofilters, and bioaerosols size distributions, rather than specifically identified pathogenic species which would have been more relevant for occupational exposure risk assessment. Literature suggests that some of the species released at the outlet may in fact have originated from within the biofilter (Martens et al., 2001; Frederickson et al., 2013) and so techniques such as this that focus more on the general microbial concentrations may miss out important trends that should be studied complementarily. It was also technically infeasible to collect inlet and outlet samples concurrently; this would have given a better prediction of bioaerosol removal. Although the time difference between inlet and outlet sample collection was minimised as far as practical, some of the bioaerosol concentration variations may be due to fluctuating levels of activities within the waste hall on a short timescale.

All pilot-scale biofilters in this study were carefully and regularly monitored to ensure they were operating optimally during the investigations, especially regarding water content and prevention of media compaction. As such the results may not allow conclusions to be drawn regarding poorly maintained systems. Although the biofilters were fed with air containing significantly high concentrations of bioaerosols, there were occasional operational interruptions (e.g. waste hall cleaning, conveyor shut down, decreased machine/vehicle activities) during sampling which could have varied the measured inlet concentrations (Stagg et al., 2013). Thus, the data presented in Table 3 as well as Figs. 4 and 5 are indicative of a high degree of variability between the replicate samples taken for each bioaerosol group at each point as shown by the error bars. This suggests that there is a high degree of measurement uncertainty, which may have led to the variable results recorded in this study. As such, the data presented here gives an insight into the likely influence of operating parameters, however further research is required to make more specific conclusions on the performance of biofilters, and particularly the mechanisms for bioaerosols removal.

\section{Conclusions}

This study shows that biofilters designed and operated for odour degradation can also achieve significant bioaerosols reduction in waste gas - 70\% (35 to 97\%) for A. fumigatus, $71 \%$ (35 to $94 \%$ ) for total fungi, $68 \%$ ( 47 to $86 \%$ ) for total mesophilic bacteria and 50 ( -4 to $85 \%$ ) for Gram negative bacteria - provided that the inlet concentration is high which is the case for most waste treatment facilities. Thus, they can be effective for the control of potentially pathogenic species in the emissions from these treatment facilities. Despite the high REs achieved, the emitted concentrations from pilot biofilters exceeded background concentrations and the EA guideline. However, from the analysis differ- 
ences may exist between the ability of the biofiltration system to deal with fungi and bacteria, as there is much more confidence with the performance for bacteria than fungi; these may be related to size differences. Furthermore, RE may deteriorate at low inlet concentration resulting in a net bioaerosol emitting potential of biofilters, and a proportion of the emitted biofilter may be originating from microbial population colonising the media surfaces, resulting in differences in species composition between contaminated process (inlet) and treated (outlet) air samples. The results also suggest that gas contact time may not play significant role in bioaerosol removal as there was no established statistical relationship over the range of EBRTs tested; however, this requires a more extensive investigation. Particle size distribution vary between the inlet and outlet air, with the outlet having predominantly greater proportion of smaller size particles that represent greater human health risk as they can penetrate the respiratory system more deeply and even to the lung alveoli where gaseous exchange occurs. However, the outlet concentrations were low, and further reduction would be achieved by the combined effect of wind dilution and dispersal as well as exposure to environmental stress from temperature, desiccation and oxygen in full scale applications. Further research with quantitative polymerase chain reaction (qPCR) and next-generation sequencing (NGS) is required to compare the species composition of both inlet and outlet air to determine whether or not new microbial populations were being emitted. Research is also required to assess the simultaneous control of odour and bioaerosols by biofilters.

\section{Research data}

The data underpinning the research presented in this paper is available at https://doi.org/10.5518/307.

\section{Acknowledgements}

This research was carried out as part of a PhD studentship funded by the University of Leeds International Research Scholarship programme. The authors are very grateful to the management and staff of Associated Waste Management Ltd, Leeds, for making their site available to conduct this pilot study.

\section{References}

Alonso, C., Zhu, X., Suidan, M.T., Kim, B.R., Kim, B.J., 1999. Mathematical model for the biodegradation of VOCs in trickle bed biofilterss. Water Sci. Technol. 39 (7), 139-146.

Álvarez-Hornos, F., Gabaldón, C., Martínez-Soria, V., Martín, M., Marzal, P., PenyaRoja, J., 2008. Biofiltration of ethylbenzene vapours: influence of the packing material. Bioresour. Technol. 99 (2), 269-276.

Instruments, Andersen, 1984. Operating Manual for the Andersen Sampler: Viable (Microbial) Particle Sizing Sampler. Andersen Samplers Inc, Atlanta, GA.

Blomquist, G., 1994. Sampling of biological particles. Analyst. 119 (1), 53-56.

Cabrol, L., Malhautier, L., Poly, F., Lepeuple, A.-S., Fanlo, J.-L., 2012. Bacterial dynamics in steady-state biofilters: beyond functional stability. FEMS Microbiol. Ecol. 79 (1), 260-271.

Calaf-Forn, M., Roca, J., Puig-Ventosa, I., 2014. Cap and trade schemes on waste management: A case study of the Landfill Allowance Trading Scheme (LATS) in England. Waste Manage. (Oxford) 34 (5), 919-928.

Chen, L., Hoff, S., 2009. Mitigating Odors from Agricultural Facilities: A Review of Literature Concerning Biofilters. Agric. Biosyst. Eng 25 (5), 751-766.

Chen, L., Hoff, S.J., 2012. A two-stage wood chip-based biofilter system to mitigate odors from a deep-pit swine building. Appl. Eng. Agric. 28 (6), 893-901.

Cornish, R., 2006. Statistics: An introduction to sample size calculations. Mathematics Learning Support Centre. [Online]. (Accessed 23 January 2017). Available from: http://www.statstutor.ac.uk/resources/uploaded/sample-size.pdf.

Dastous, P.A., Soreanu, G., Nikiema, J., Heitz, M., 2005. Biofiltration of three alcohols on a mature bed compost. Air \& Water Management Association, Pittsburgh, PA.

Devinny, J.S., Deshusses, M.A., Webster, T.S., 1999. Biofiltration for Air Pollution Control. Lewis Publishers, Florida.

Douwes, J., Thorne, P., Pearce, N., Heederik, D., 2003. Bioaerosol health effects and exposure assessment: progress and prospects. Ann. Occup. Hyg. 47 (3), 187 200
Eduard, W., Heederik, D., Duchaine, C., Green, B.J., 2012. Bioaerosol exposure assessment in the workplace: the past, present and recent advances. J. Environ. Monit. 14 (2), 334-339.

Elias, A., Barona, A., Arreguy, A., Rios, J., Aranguiz, I., Peñas, J., 2002. Evaluation of a packing material for the biodegradation of $\mathrm{H}_{2} \mathrm{~S}$ and product analysis. Process Biochem. 37 (8), 813-820.

Environment Agency, 2010. Composting and potential health effects from bioaerosols: our interim guidance for permit applicants. Position Statement [Online]. (Accessed 14 May 2018). Available from: http://www.organicsrecycling.org.uk/uploads/article1822/Composting_\&_bioaerosols_position_statement_fina_2010l\%5B1\%5D.pdf.

Environment Agency, 2017. Environmental monitoring of bioaerosols at regulated facilities. Technical Guidance Note (Monitoring) M9, January 2017, version 1.

Environment Agency, 2018. Guidance - Bioaerosol monitoring at regulated facilities - use of M9: RPS 209. [Online]. (Accessed 12 August). Available from: https:/ www.gov.uk/government/publications/bioaerosol-monitoring-at-regulatedfacilities-use-of-m9-rps-209/bioaerosol-monitoring-at-regulated-facilitiesuse-of-m9-rps-209.

Ferguson, R.M.W., Neath, C.E., Dumbrell, A.J., Whitby, C. and Colbeck, I., 2017. Novel insights into the size distribution of bacterial bioaerosols at composting sites In: Focus Meeting 10 - Bioaerosols, 8 June 2017, University of Bristol, UK. North Somerset: The Aerosol Society, pp. 25-28.

Fletcher, L.A., Jones, N., Warren, L., Stentiford, E.I., 2014. Understanding biofilter performance and determining emission concentrations under operational conditions. Sniffer, Edinburgh, Scotland.

Frederickson, J., Boardman, C.P., Gladding, T.L., Simpson, A.E., Howell, G., Sgouridis, F., 2013. Evidence: Biofilter performance and operation as related to commercial composting. Environment Agency, Bristol.

Fulazzaky, M.A., Talaiekhozani, A., Ponraj, M., Abd Majid, M., Hadibarata, T., Goli, A. 2014. Biofiltration process as an ideal approach to remove pollutants from polluted air. Desalin. Water Treat. 52 (19-21), 3600-3615.

Gao, L., Keener, T., Zhuang, L., Siddiqui, K., 2001. A technical and economic comparison of biofiltration and wet chemical oxidation (scrubbing) for odor control at wastewater treatment plants. Environ. Eng. Policy 2 (4), 203-212.

Hambach, R., Droste, J., François, G., Weyler, J., Van Soom, U., De Schryver, A. Vanoeteren, J., van Sprundel, M., 2012. Work-related health symptoms among compost facility workers: a cross-sectional study. Arch. Publ. Health 70 (1), p13.

Hellenbrand, K.E., Reade, A.E., 1992. Microorganisms associated with fuel wood chips and their impact on indoor air quality: a review. Int. Biodeterior Biodegrad. 29 (1), 19-43.

Holland, C., 2011. AWM's new recycling facility's power plan. Bradford Telegraph and Argus [Online]. (Accessed 6 June 2015). Available from: http:// www.thetelegraphandargus.co.uk/business/9326078.AWM_s_new_recycling_ facility_s_power_plan/.

Hurst, C.J., Crawford, R.L., Garland, J.L., Lipson, D.A., 2007. Manual of environmental microbiology. American Society for Microbiology Press.

Husman, T., 1996. Health effects of indoor-air microorganisms. Scand. J. Work Environ. Health, 5-13.

Janni, K.A., Nicolai, R.K., Hoff, S. and Stenglein, R.M. 2011. Air Quality Education. In: Animal Agriculture: Biofilters for Odor and Air Pollution Mitigation in Animal Agriculture. Agricultural and Biosystems Engineering Extension and Outreach Publications. Book 3. pp. 1-9.

Jorio, H., Bibeau, L., Viel, G., Heitz, M., 2000. Effects of gas flow rate and inlet concentration on xylene vapors biofiltration performance. Chem. Eng. J. 76 (3), 209-221.

Juteau, P., Rho, D., Larocque, R., LeDuy, A., 1999. Analysis of the relative abundance of different types of bacteria capable of toluene degradation in a compost biofilter. Appl. Microbiol. Biotechnol. 52 (6), 863-868.

Kafle, G.K., Chen, L., Neibling, H., Brian He, B., 2015. Field evaluation of wood barkbased down-flow biofilters for mitigation of odor, ammonia, and hydrogen sulfide emissions from confined swine nursery barns. J. Environ. Manage. 147, $164-174$.

Kell, D.B., Kaprelyants, A.S., Weichart, D.H., Harwood, C.R., Barer, M.R., 1998. Viability and activity in readily culturable bacteria: a review and discussion of the practical issues. Antonie van Leeuwenhoek 73 (2), 169-187.

Kummer, V., Thiel, W.R., 2008. Bioaerosols-sources and control measures. Int. J. Hyg. Environ. Health 211 (3-4), 299-307.

Lee, C., Lin, S., 2007. Handbook of Environmental Engineering Calculations. McGraw-Hill Prof Med/Tech.

Leson, G., Winer, A.M., 1991. Biofiltration: an innovative air pollution control technology for VOC emissions. J. Air Waste Manage. Assoc. 41 (8), 1045-1054.

Liu, Q., Li, M., Chen, R., Li, Z., Qian, G., An, T., Fu, J., Sheng, G., 2009. Biofiltration treatment of odors from municipal solid waste treatment plants. Waste Manage. 29 (7), 2051-2058.

Lu, C., Lin, M.-R., Chu, C., 2002. Effects of pH, moisture, and flow pattern on tricklebed air biofilter performance for BTEX removal. Adv. Environ. Res. 6 (2), 99-106.

Macher, J.M., 1989. Positive-hole correction of multiple-jet impactors for collecting viable microorganisms. Am. Indus. Hyg. Assoc. J. 50 (11), 561-568.

Malmros, P., Sigsgaard, T., Bach, B., 1992. Occupational health problems due to garbage sorting. Waste Manage. Res. 10 (3), 227-234.

Martens, W., Martinec, M., Zapirain, R., Stark, M., Hartung, E., Palmgren, U., 2001. Reduction potential of microbial, odour and ammonia emissions from a pig facility by biofilters. Int. J. Hyg. Environ. Health 203 (4), 335-345.

Meka, V., Van Oostrom, J., 2004. Bellows-less lung system for the human patient simulator. Med. Biol. Eng. Comput. 42 (3), 413-418. 
Menetrez, M., Foarde, K., Esch, R., Schwartz, T., Dean, T., Hays, M., Cho, S. Betancourt, D., Moore, S., 2009. An evaluation of indoor and outdoor biological particulate matter. Atmos. Environ. 43 (34), 5476-5483.

Millner, P., Marsh, P., Snowden, R., Parr, J., 1977. Occurrence of Aspergillus fumigatus during composting of sewage sludge. Appl. Environ. Microbiol. 34 (6), 765-772.

Nasir, Z.A., Tyrrel, S., 2017. Advances in bioaerosols emissions characterization from green waste composting. In: Focus Meeting 10 - Bioaerosols, 8 June 2017 , University of Bristol, UK. North Somerset: The Aerosol Society, pp. 12-15.

Nicolai, R., Janni, K., 2001a. Biofilter media mixture ratio of wood chips and compost treating swine odors. Water Sci. Technol. 44 (9), 261-267.

Nicolai, R.E., Janni, K.A., 2001. Determining pressure drop through compost-wood chip biofilter Media. ASAE Paper No. 014080. St. Joseph, Mich.: ASAE.

Ottengraf, S., Konings, J., 1991. Emission of microorganisms from biofilters. Bioprocess. Eng. 7 (1-2), 89-96.

Pagella, C., De Faveri, D.M., 2000. $\mathrm{H}_{2} \mathrm{~S}$ gas treatment by iron bioprocess. Chem. Eng. Sci. 55 (12), 2185-2194.

Pankhurst, L.J., Deacon, L.J., Liu, J., Drew, G.H., Hayes, E.T., Jackson, S., Longhurst, P.J. Longhurst, J.W.S., Pollard, S.J.T., Tyrrel, S.F., 2011. Spatial variations in airborne microorganism and endotoxin concentrations at green waste composting facilities. Int. J. Hyg. Environ. Health 214 (5), 376-383.

Parry, G., 2018. Bioaerosol Risk Assessment - Alne Material Recycling Facility. [Online]. (Accessed 12 June 2018). Available from: https://consult. environment-agency.gov.uk/psc/yo61-1tu-allium-helmsleyItd/supporting_documents/9f\%20Supporting\%20Documents\%20\%20Bioaerosol\% 20risk\%20assessment.pdf.

Pearson, C., Littlewood, E., Douglas, P., Robertson, S., Gant, T.W., Hansell, A.L., 2015. Exposures and health outcomes in relation to bioaerosol emissions from composting facilities: a systematic review of occupational and community studies. J. Toxicol. Environ. Health, Part B 18 (1), 43-69.

Quanjer, P.H., Tammeling, G., Cotes, J., Pedersen, O., Peslin, R., Yernault, J., 1993. Lung volumes and forced ventilatory flows. Eur Respiratory Soc.

Quigley, C., Easter, C., Burrowes, P., Witherspoon, J., 2004. Biotechnology-based odour control: design criteria and performance data. Water Sci. Technol. 50 (4), 319-326.

Rabe, R., Becker, M., 2000. Emissionen von Pilzsporen aus Biofiltern von Kompostwerken. Gefahrstoffe-Reinhaltung der Luft. 60, 168-170.

Ralebitso-Senior, T.K., Senior, E., Di Felice, R., Jarvis, K., 2012. Waste gas biofiltration: advances and limitations of current approaches in microbiology. Environ. Sci. Technol. 46 (16), 8542-8573.

Sanchez-Monedero, M.A., Stentiford, E.I., Mondini, C., 2003. Biofiltration at composting facilities: effectiveness for bioaerosol control. Environ. Sci. Technol. 37 (18), 4299-4303.

Schlegelmilch, M., Herold, T., Streese, J., Hensel, A., Stegmann, R., 2005. The potential to reduce emissions of airborne microorganisms by means of biological waste gas treatment systems. Waste Manage. 25 (9), 955-964.

Schnelle, K.B., Brown, C.A., 2002. Air pollution control technology handbook. thesis CRC Press LLC.
Searl, A., 2008. Exposure-response relationship for bioaerosols emissions from waste treatment processes. IOM, Edinburgh.

Stagg, S., Bowry, A., Kelsey, A., Crook, B., 2010. Bioaerosol emissions from waste composting and the potential for workers' exposure. RR786. Derbyshire: Health and Safety Executive.

Stagg, S., Sandys, V., Crook, B., Wood, J., McAlinden, J., 2013. Occupational Hygiene implications of processing waste at Materials Recycling Facilities (MRFs). [Online]. (Accessed 30 August). Available from: http://www.hse.gov.uk/ research/rrpdf/rr977.pdf.

Surrey County Council, 2017. Types of Waste Management Facilities: An Explanation Note. [Online]. (Accessed 20 June 2018). Available from: https:// www.surreycc.gov.uk/_data/assets/pdf_file/0010/147367/2017-10-27-Typesof-waste-mgt-facilties-explanation-note.pdf.

Swan, J., Kelsey, A., Crook, B., Gilbert, E., 2003. Occupational and environmental exposure to bioaerosols from composts and potential health effects: a critical review of published data. HSE Books.

Taha, M., Pollard, S.J., Sarkar, U., Longhurst, P.J., 2004. The influence of process variables on bioaerosol emission flux and exposure-estimating fugitive bioaerosol releases from static compost windrows. Proceedings of the 1st UK Conference and Exhibition on Biodegradable and Residual Waste Management.

TSI Incorporated, 2013. Health-Based Particle-Size-Selective Sampling. [Online]. (Accessed 21 August 2017). Available from: http://www.tsi.com/uploadedFiles/ Site_Root/Products/Literature/Application_Notes/ITI-050.pdf.

Tymczyna, L., Chmielowiec-Korzeniowska, A., Raczynska, J., Drabik, A., 2011. Removal of microbial contaminants from pig house air using biofilter organic media. Ann. Anim. Sci. 11, 453-464.

Valter Francescato, E.A., Bergomi, L.Z., Metschina, C., Schnedl, C., Krajnc, N., Kajetan Koscik, P.G., Nocentini, G., Stranieri, S., 2008. Wood Fuels Handbook: Production, Quality Requirements, Trading. AIEL - Italian Agriforestry Energy Association, Italy.

van Kampen, V., Deckert, A., Hoffmeyer, F., Taeger, D., Brinkmann, E., Brüning, T., Raulf-Heimsoth, M., Bünger, J., 2012. Symptoms, spirometry, and serum antibody concentrations among compost workers exposed to organic dust. J. Toxicol. Environ. Health, Part A 75 (8-10), 492-500.

Varley, N., 2013. Technical Assessment Odour Abatement System for AWM Gelderd Road. Unpublished.

Wani, A.H., Branion, R.M.R., Lau, A.K., 1997. Biofiltration: A promising and costeffective control technology for Odors, VOCs and air toxics. J. Environ. Sci. Health. Part A: Environ. Sci. Eng. Toxicol. 32 (7), 2027-2055.

Willeke, K., Oian, Y., Donnelly, J., Grinshpun, S., Ulevicius, V., 1996. Penetration of airborne microorganisms through a surgical mask and a dust/mist respirator. Am. Indus. Hyg. Assoc. 57 (4), 348-355.

Williams, M., Lamarre, B., Butterfield, D., Tyrrel, S., Longhurst, P., Drew, G., AlAshaab, R., Nelson, A., Gladding, T. Simpson, A., Coughlin, D., Hansell, A., 2013. Bioaerosols and odour emissions from composting facilities Defra Project WR 1121. Department Environ. Food Rural Affairs.

Yang, Y., Allen, E.R., 1994. Biofiltration control of hydrogen sulfide 1. Design and operational parameters. Air Waste 44 (7), 863-868. 